\title{
Extracellular Biofabrication, Characterization, and Antimicrobial Efficacy of Silver Nanoparticles Loaded on Cotton Fabrics Using Newly Isolated Streptomyces sp. SSHH-1E
}

\author{
Noura El-Ahmady El-Naggar, ${ }^{1}$ Attiya Mohamedin, ${ }^{2}$ \\ Sarah Shawqi Hamza, ${ }^{2}$ and Abdel-Dayem Sherief ${ }^{2}$ \\ ${ }^{1}$ Department of Bioprocess Development, Genetic Engineering and Biotechnology Research Institute, City of Scientific Research and \\ Technological Applications, Alexandria 21934, Egypt \\ ${ }^{2}$ Department of Botany, Faculty of Science, Mansoura University, Mansoura 35516, Egypt
}

Correspondence should be addressed to Noura El-Ahmady El-Naggar; nouraalahmady@yahoo.com

Received 19 February 2016; Accepted 3 April 2016

Academic Editor: Xuping Sun

Copyright (C) 2016 Noura El-Ahmady El-Naggar et al. This is an open access article distributed under the Creative Commons Attribution License, which permits unrestricted use, distribution, and reproduction in any medium, provided the original work is properly cited.

\begin{abstract}
Biological method for silver nanoparticles synthesis has been developed to obtain cost effective, clean, nontoxic, and ecofriendly size-controlled nanoparticles. The objective of this study is extracellular biosynthesis of antimicrobial AgNPs using cell-free supernatant of a local Streptomyces sp. strain SSHH-1E. Different medium composition and fermentation conditions were screened for maximal AgNPs biosynthesis using Plackett-Burman experimental design and the variables with statistically significant effects were selected to study their combined effects and to find out the optimum values using a Box-Behnken design. The synthesized AgNPs were characterized using UV-visible spectroscopy, transmission electron microscopy, Fourier transform infrared spectroscopy, and energy dispersive X-ray spectroscopy. Rapid biosynthesis of AgNPs was achieved by addition of $1 \mathrm{mM}$ $\mathrm{AgNO}_{3}$ solution to the cell-free supernatant. The produced particles showed a single surface plasmon resonance peak at $400 \mathrm{~nm}$ by UV-Vis spectroscopy which confirmed the presence of AgNPs. Streptomyces sp. SSHH-1E was identified as Streptomyces narbonensis SSHH-1E. Transmission electron microscopy study indicated that the shape of AgNPs is spherical and the size is ranging from 20 to $40 \mathrm{~nm}$. Fourier transform infrared spectroscopy analysis provides evidence for proteins as possible reducing and capping agents. Furthermore, the biosynthesized AgNPs significantly inhibited the growth of medically important pathogenic Gram-positive and Gram-negative bacteria and yeast. The maximum biosynthesis of AgNPs was achieved at initial $\mathrm{pH}$ of 8, peptone of $0.5 \mathrm{~g}$, and inoculum age of $48 \mathrm{~h}$. The statistical optimization resulted in a 4.5-fold increase in the production of AgNPs by Streptomyces narbonensis SSHH-1E.
\end{abstract}

\section{Introduction}

Nanotechnology involves the production, manipulation, and use of materials ranging in size from less than a micron to that of individual atoms. An important aspect of nanotechnology is the development of toxicity-free synthesis of metal nanoparticles which is a great challenge. Silver, in its metallic as well as ionic forms, exhibits cytotoxicity against several microorganisms and hence can be used as antimicrobial agent [1].

Silver nanoparticles (AgNPs) have been widely employed in various fields due to their physicochemical properties including extremely small size and large surface area relative to their volume [2]. The antimicrobial activity of AgNPs has now been well established and they are confirmed to possess antifungal, anti-inflammatory, antiviral, antiangiogenic, and antipermeability activities [3]. AgNPs can be used in medicine to reduce infections as well as to prevent bacteria colonization on prostheses [4], as antimicrobial agents in surgically implanted catheters in order to reduce the infections caused during surgery [5], vascular grafts [6], dental materials [7], and stainless steel materials and human skin [8]. In the last few decades there has been increased interest in reducing 
the availability of commercial textile containing antibacterial agents due to environmental pollution. Since silver is a good antibacterial agent and nontoxic and natural inorganic metal, it is used in different kinds of textile fibers. AgNPs are also used in hygienic products including water purification systems, linings of washing machine, dishwashers, refrigerators, and toilet seats [9]. AgNPs could have many applications in cosmetics, microelectronics, conductive inks, adhesives [10], nonlinear optics, spectrally selective coating for solar energy absorption, biolabeling, intercalation materials for electrical batteries, catalysis in chemical reactions [11], high-sensitivity biomolecular detection and diagnostics [12], antimicrobials [13], therapeutics [14], silver nanocoated medical devices [15], and optical receptors [16].

Now AgNPs are commonly synthesized by chemical reduction [17], thermal treatment [18], irradiation [19], and laser ablation [20], which are of low yield, energy-intensive, difficult to scale up, and often producing high levels of hazardous wastes and may require the use of organic solvents and toxic reducing agents. So, these techniques yield extremely expensive materials. In addition, the produced nanoparticles exhibit undesirable aggregation with time. Green synthesis methods, involving organisms ranging from bacteria to fungi and even plants, have been developed for the synthesis of these nanoparticles which eliminate the use of toxic chemicals during their synthesis process and to obtain sustainable, cost effective, clean, nontoxic, easily scaled up for large scale synthesis, and ecofriendly size-controlled nanoparticles [21]. Microbial properties of bioaccumulation, biosorption, biodetoxification, and biomineralization have been regarded as opportunity to use them as nanofactories for mining nanomaterials $[22,23]$. Various bacteria, yeast, and fungi are known to synthesize silver nanoparticles production [24, 25]. The produced nanoparticles have different sizes and shapes. Nanoparticles resulting from some microbial processes are composite materials and consist of inorganic component and special organic matrix such as proteins, lipids, or polysaccharides and they have unique chemical and physical properties which are different from the properties of conventionally produced nanoparticles and of other microorganisms even when they are incubated in the same medium under the same conditions [26]. So, it is important to examine new classes of microbes to synthesize silver nanoparticles with technologically important properties. Synthesis of nanoparticles using microorganisms can potentially eliminate this problem by making the nanoparticles more biocompatible. Silver nanoparticles have been successfully synthesized using various bacteria [27], fungi [28], actinomycetes [29], and plant extracts [30].

The classical optimization strategy used is one-variableat-a-time (OVAT) optimization, where each parameter is optimized by changing it at the same time while the other factors were maintained at a constant level [31]. Time consumption, requirement of more experimental data sets [32], and missing the interactions among parameters are the obstacles in predicting the accurate results when the conventional optimization procedures like "one-variable-at-a-time" were applied [33]. Statistical optimization has become a common practice in biotechnology. It has the advantages of taking into account the interaction among the nutrients, is less time consuming, and avoids the erroneous interpretation occurring in one-factor-at-a-time optimization [34].

There are many available experimental design strategies, including full-factorial Plackett-Burman; it is often used when more than five independent variables are being investigated. The disadvantage of the Plackett-Burman design is that it only allows first-order effects and ignores potential interactions [35]. Response surface methodology (RSM) was first described in [36] as an experimental strategy for obtaining optimum conditions of a multivariable system. Process optimization using RSM usually involves simultaneous testing of many factors in a limited number of experiments. Therefore, RSM takes less time and effort than the traditional approach. This method quantifies possible interactions between various factors.

The aim of this study was to apply the Plackett-Burman design, followed by RSM to optimize the culture medium composition and environmental factors for AgNPs production by Streptomyces sp. SSHH-1E, to characterize the green synthesized AgNPs, and to evaluate the antimicrobial activities of cotton fabrics coated with silver nanoparticles.

\section{Materials and Methods}

2.1. Microorganism and Cultural Conditions. Streptomyces spp. used in this study were isolated from various soil samples collected from different localities of Egypt. Actinomycetes from the soil had been isolated using standard dilution plate method procedure on Petri plates containing starch nitrate agar medium of the following composition $(\mathrm{g} / \mathrm{L})$ : starch, 20; $\mathrm{KNO}_{3}, 2 ; \mathrm{K}_{2} \mathrm{HPO}_{4}, 1 ; \mathrm{MgSO}_{4} \cdot 7 \mathrm{H}_{2} \mathrm{O}, 0.5 ; \mathrm{NaCl}, 0.5$; $\mathrm{CaCO}_{3}, 3 ; \mathrm{FeSO}_{4} \cdot 7 \mathrm{H}_{2} \mathrm{O}, 0.01$; agar, 20 . Nystatin $(50 \mu \mathrm{g} / \mathrm{mL})$ was incorporated as an antifungal agent to minimize fungal contamination. Then plates were incubated for 7 days at $30^{\circ} \mathrm{C}$. The actinomycetes strains predominant on media were picked out, purified, and maintained on starch nitrate agar slants. These strains were stored as spore suspensions in $20 \%(\mathrm{v} / \mathrm{v})$ glycerol at $-20^{\circ} \mathrm{C}$ for subsequent investigation.

2.2. Preparation of Inoculum. To prepare the filtrate for biosynthesis studies, the Streptomyces sp. SSHH-1E was grown aerobically in $50 \mathrm{~mL}$ liquid broth containing starch $20 \mathrm{~g}$; $\mathrm{NaNO}_{3} 2 \mathrm{~g} ; \mathrm{K}_{2} \mathrm{HPO}_{4} 1 \mathrm{~g} ; \mathrm{MgSO}_{4} \cdot 7 \mathrm{H}_{2} \mathrm{O} 0.5 \mathrm{~g}$; and distilled water up to $1 \mathrm{~L}$. The culture flasks were inoculated with three disks of $9 \mathrm{~mm}$ diameter taken from 7-day-old stock culture grown on starch nitrate agar medium and incubated on an orbital shaker $(150 \mathrm{rpm})$ for $48 \mathrm{~h}$ at $28^{\circ} \mathrm{C}$ and then used as inoculum for subsequent experiments.

2.3. Extracellular Synthesis of AgNPs. In order to screen an efficient strain for the synthesis of AgNPs, 40 actinomycetes strains were freshly inoculated in 250 Erlenmeyer flasks containing $50 \mathrm{~mL}$ of the production medium consisting of (g/L) soluble starch 20; $\mathrm{NaNO}_{3} 2 ; \mathrm{K}_{2} \mathrm{HPO}_{4} 1 ; \mathrm{MgSO}_{4} \cdot 7 \mathrm{H}_{2} \mathrm{O}$ 0.5 ; and distilled water up to $1 \mathrm{~L}$. The inoculated flasks were incubated on a rotatory incubator shaker at $30^{\circ} \mathrm{C}$ and $150 \mathrm{rpm}$ for 5 days. After incubation period, the cell-free supernatant 
was obtained by centrifugation at $5000 \mathrm{rpm}$ for $30 \mathrm{~min}$. For the biosynthesis of AgNPs to occur, 1\% (v/v) of $1 \mathrm{mM} \mathrm{AgNO}_{3}$ was added to cell-free supernatant and incubated, under dark condition, on an orbital shaker for $24 \mathrm{~h}$ at $30^{\circ} \mathrm{C}$.

The cell-free supernatant without addition of $\mathrm{AgNO}_{3}$ was maintained as a control. Then the reaction mixture was centrifuged at $10000 \mathrm{rpm}$ for $15 \mathrm{~min}$ for disposing any impurities and the bioreduction reaction was monitored by visual color change and UV-visible absorbance of the reaction mixture in the $300-600 \mathrm{~nm}$ range. Based on the rapid reduction of $\mathrm{AgNO}_{3}$ into AgNPs a proficient Streptomyces strain was selected and used for further characterization.

2.4. Identification of Streptomyces sp. SSHH-1E. The isolate was identified on the basis of morphological, biochemical, and physiological characteristics following the methods of Shirling and Gottlieb [37]. The isolate was further identified according to $16 \mathrm{~S}$ rRNA gene sequencing studies.

2.5. 16S rRNA Sequencing. The preparation of genomic DNA of the strain was conducted in accordance with the methods described in [38]. The PCR amplification reaction was performed in a total volume of $100 \mu \mathrm{L}$, which contained $1 \mu \mathrm{L}$ DNA, $10 \mu \mathrm{L}$ of $250 \mathrm{mM}$ deoxyribonucleotide $5^{\prime}$-triphosphate (dNTP), $10 \mu \mathrm{L}$ PCR buffer, $3.5 \mu \mathrm{L} 25 \mathrm{mM} \mathrm{MgCl}_{2}$ and $0.5 \mu \mathrm{L}$ Taq polymerase, and $4 \mu \mathrm{L}$ of $10 \mathrm{pmol}$ (each) forward $16 \mathrm{~S}$ rRNA primer $27 f\left(5^{\prime}\right.$-AGAGTTTGATCMTGCCTCAG- $\left.3^{\prime}\right)$ and reverse $16 \mathrm{~S}$ rRNA primer $1492 \mathrm{r}\left(5^{\prime}\right.$-TACGGYTACCTTGTTACGACTT- $3^{\prime}$ ) and water was added up to $100 \mu \mathrm{L}$. The PCR-apparatus was programmed as follows: 5 min denaturation at $94^{\circ} \mathrm{C}$, followed by 35 amplification cycles of $1 \mathrm{~min}$ at $94^{\circ} \mathrm{C}, 1 \mathrm{~min}$ of annealing at $55^{\circ} \mathrm{C}$, and $2 \mathrm{~min}$ of extension at $72^{\circ} \mathrm{C}$, followed by a $10 \mathrm{~min}$ final extension at $72^{\circ} \mathrm{C}$. The PCR mixture was then analyzed via agarose gel electrophoresis, and the remaining mixture was purified using QIA quick PCR purification reagents (Qiagen, USA). The purified PCR product was sequenced by using two primers, 518F, $5^{\prime}$-CCA GCA GCC GCG GTA ATA CG-3', and 800R, $5^{\prime}$-TAC CAG GGT ATC TAA TCC- $3^{\prime}$. Sequencing was performed by using BigDye terminator cycle sequencing kit (Applied Biosystems, USA). Sequencing product was resolved on Applied Biosystems model 3730XL automated DNA sequencing system (Applied Biosystems, USA) and deposited in the GenBank database under accession number KJ676475.

2.6. Sequence Alignment and Phylogenetic Analysis. The complete $16 \mathrm{~S}$ rRNA gene sequence of strain SSHH-1E was aligned with the corresponding $16 \mathrm{~S}$ rRNA sequences of the type strains of representative members of the genus Streptomyces retrieved from the GenBank, EMBL, DDBJ, and PDB databases by using BLAST programme (https://blast.ncbi.nlm .nih.gov/Blast.cgi?PAGE_TYPE=BlastSearch) [39] and the software package MEGA4 version 2.1 [40] was used for multiple alignment and phylogenetic analysis. The phylogenetic tree was constructed via the bootstrap test of neighbourjoining algorithm [41] based on the 16S rRNA gene sequences of strain SSHH-1E and related organisms.
2.7. Identifying the Significant Variables Using Plackett-Burman Design (PBD). Screening process was carried out by conducting the experiments to determine which variables significantly affect AgNPs production. A total of 14 independent (assigned) and 5 unassigned variables (commonly referred to as dummy variables) were screened in PlackettBurman experimental design of 20 trials. Dummy variables $\left(D_{1}, D_{2}, D_{3}, D_{4}\right.$, and $\left.D_{5}\right)$ are used to estimate experimental errors in data analysis. All experiments were carried out in duplicate and average of AgNPs biosynthesized was taken as the response. Based on the AgNPs biosynthesized, the factorial experiment was analyzed using regression analysis and ANOVA. From the regression analysis, the variables, which were significant $(P<0.1)$, were considered to have greater impact on the AgNPs biosynthesized and were further optimized by Box-Behnken design.

Plackett-Burman experimental design [35] is based on the first-order model

$$
Y=\beta_{0}+\sum \beta_{i} X_{i}
$$

where $Y$ is the response variable (AgNPs biosynthesis), $\beta_{0}$ is the model intercept and $\beta_{i}$ is the linear coefficient, and $X_{i}$ is the level of the independent variable.

2.8. Box-Behnken Design (BBD). Three variables Box-Behnken design for response surface methodology was used to study the combined effect of inoculum age, $\mathrm{pH}$, and peptone on silver nanoparticles synthesis over three levels. In this study, the experimental plan consisted of 15 trials and the independent variables were studied at three different levels, low (-1), medium (0), and high (+1). The experimental results of RSM were fitted via the response surface regression procedure using the following second-order polynomial equation:

$$
Y=\beta_{0}+\sum_{i} \beta_{i} X_{i}+\sum_{i i} \beta_{i i} X_{i}^{2}+\sum_{i j} \beta_{i j} X_{i} X_{j}
$$

In the equation above $Y$ is the predicted response, $\beta_{0}$ is the regression coefficients, $\beta_{i}$ is the linear coefficient, $\beta_{i i}$ is the quadratic coefficients, $\beta_{i j}$ is the interaction coefficients, and $X_{i}$ is the coded levels of independent variables. However, in this study, the independent variables were coded as $X_{1}, X_{2}$, and $X_{3}$. Thus, the second-order polynomial equation can be presented as follows:

$$
\begin{aligned}
Y= & \beta_{0}+\beta_{1} x_{1}+\beta_{2} x_{2}+\beta_{3} x_{3}+\beta_{12} x_{1} x_{2}+\beta_{13} x_{1} x_{3} \\
& +\beta_{23} x_{2} x_{3}+\beta_{11} x_{1}^{2}+\beta_{22} x_{2}^{2}+\beta_{33} x_{3}^{2} .
\end{aligned}
$$

\subsection{Characterization of Silver Nanoparticles}

2.9.1. UV-Visible Spectral Analysis. The reduction of metallic $\mathrm{Ag}^{+}$ions was monitored by measuring the UV-Vis spectrum after 24 hours of reaction. A small aliquot was drawn from the reaction mixture and a spectrum was taken on a wavelength from 300 to $600 \mathrm{~nm}$ on UV-Vis spectrophotometer (Libra S30/30PC). 


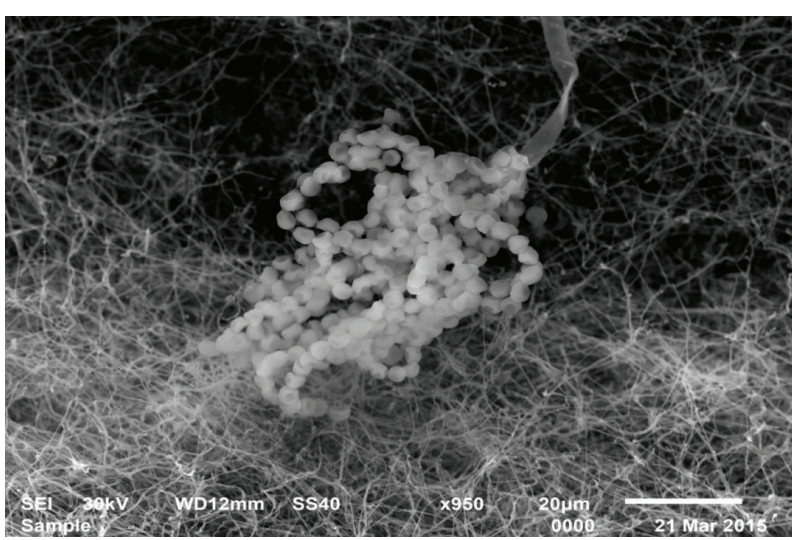

(a)

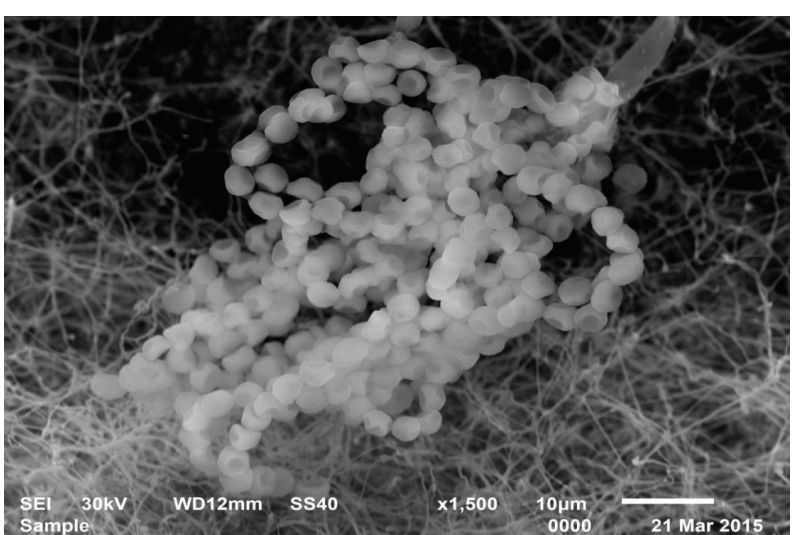

(b)

FIGURE 1: Scanning electron micrograph showing the spore chain morphology and spore surface ornamentation of Streptomyces sp. SSHH-1E grown on starch nitrate agar medium for 14 days at $30^{\circ} \mathrm{C}$ at magnification of $950 \mathrm{x}$ (a) and 1500x (b).

2.9.2. Transmission Electron Microscopy (TEM) Analysis. The morphology and crystal structure of the AgNPs were studied using high resolution transmission electron microscopy (HRTEM) and selected-area electron diffraction (SAED) (JEOL-JEM-100 CXII instrument), by drying a drop of the washed colloidal dispersion onto a copper grid covered with a conductive polymer. The size and shape of AgNPs synthesized using Streptomyces narbonensis SSHH-1E were visualized using $200 \mathrm{kV}$ ultrahigh resolution TEM.

2.9.3. Energy Dispersive X-ray (EDX) Spectroscopy. The nanoparticles were mounted on the copper stubs, and the images were studied using scanning electron microscope (Hitachi, Model S-3400N) with secondary electron detectors at an operating voltage of $20 \mathrm{kV}$ and for elemental analysis energy dispersive X-ray (EDX) analysis was done in Noran-System Six X-ray microanalysis system (Thermo Electron Corporation, USA) coupled to scanning electron microscope (SEM).

2.9.4. Fourier Transform Infrared (FTIR) Spectroscopy. The bioreduced $\mathrm{AgNO}_{3}$ solution was centrifuged at 10,000 rpm for $15 \mathrm{~min}$ and the freeze dried sample was grinded with $\mathrm{KBr}$ pellets used for FTIR measurements. The spectrum was recorded in the range of $4000-500 \mathrm{~cm}^{-1}$. The FTIR spectrum of samples was recorded on FTIR instrument (Shimadzu FTIR-8400 S).

2.10. Antimicrobial Activity of AgNPs Suspension Loaded Cotton Fabrics by Disc Diffusion Method. According to Durán et al. [42], cotton fabrics were washed, sterilized, and dried before use. AgNPs were loaded onto the surface of cotton fabrics with maximum dimensions of $1 \mathrm{~cm} \times 1 \mathrm{~cm}$, followed by air drying. The antimicrobial activity of AgNPs treated cotton fabrics was evaluated against bacterial pathogens of Grampositive (Staphylococcus aureus) and Gram-negative bacteria (E. coli) and yeast (Candida albicans) on Luria Bertani (LB) agar plates at different time intervals. Cell-free supernatant without silver AgNPs was used as a control. The plates were incubated at $30^{\circ} \mathrm{C}$ for $24 \mathrm{~h}$ and were then examined for the presence of zones of inhibition.

2.11. Stability and Dispersibility of Synthesized Silver Nanoparticles. The stability and dispersibility of synthesized silver nanoparticles were monitored for a long time period after their synthesis by UV-visible spectroscopy and transmission electron microscopy. The AgNPs were stored in closed glass vials under dark conditions. Afterwards, samples were taken at different predefined times (e.g., 1 day, 1 month, 2 months, 4 months, and 6 months) and were characterized by UV-visible spectroscopy, transmission electron microscopy to check the changes in the surface plasmon bands, morphology, and distribution.

\section{Results and Discussion}

3.1. Isolation and Screening of Actinomycetes for Extracellular Synthesis of AgNPs. Among 40 different actinomycetes strains which were isolated and screened for biological synthesis of AgNPs, Streptomyces sp. SSHH-1E was found to exhibit rapid and high ability in AgNPs biosynthesis. The appearance of dark brown color in the filtrate after reaction with the $\mathrm{Ag}^{+}$ions is a clear indicator of the reduction of metal ions and formation of AgNPs. The pale yellow colored reaction mixture turned into a dark brown color indicating the formation of AgNPs due to excitation of surface plasmon vibrations in the nanoparticles [43].

\subsection{Biological Characteristics and Identification of Strepto-} myces sp. SSHH-1E. The strain has a Rectiflexibiles spore type carrying more than 50 smooth-surfaced spores with highly branched substrate mycelium without fragmentation. No sporangia are observed (Figure 1), and the aerial mycelium is gray on standard media (Table 1). Melanin pigments are not produced on peptone yeast iron agar, tryptone yeast broth, and tyrosine agar. The strain intensively utilizes $\mathrm{D}(+)$ galactose, $\mathrm{D}(+)$-xylose, mannose, ribose, and arabinose and weakly utilizes $\mathrm{D}(+)$-glucose, $\mathrm{D}(+)$-fructose, and trehalose. 
TABLE 1: Culture properties of Streptomyces sp. SSHH-1E.

\begin{tabular}{|c|c|c|c|c|}
\hline Medium & Color of aerial mycelium & Color of substrate mycelium & Diffusible pigment & Growth \\
\hline ISP medium 2 (yeast extract, malt extract agar) & Gray & Brown & Nonpigmented & Excellent \\
\hline ISP medium 3 (oatmeal agar) & Gray & Dark gray & Nonpigmented & Very good \\
\hline ISP medium 4 (inorganic salt starch agar) & Gray & Dark brown & Nonpigmented & Excellent \\
\hline ISP medium 5 (glycerol asparagine agar) & White & Yellowish white & Nonpigmented & Weak \\
\hline ISP medium 6 (peptone yeast extract iron agar) & No sporulation & No distinctive pigments & Nonpigmented & Very weak \\
\hline ISP medium 7 (tyrosine agar) & No sporulation & No distinctive pigments & Nonpigmented & Very weak \\
\hline
\end{tabular}

TABLE 2: Comparison of biological properties of Streptomyces sp. SSHH-1E and other related Streptomyces species.

\begin{tabular}{|c|c|c|}
\hline Characteristics & Streptomyces sp. SSHH-1E & Streptomyces narbonensis \\
\hline Aerial mycelium on ISP medium 2 & Gray & Gray \\
\hline Substrate mycelium on ISP medium 2 & Brown & Greenish yellow \\
\hline Production of diffusible pigment & None & None \\
\hline Spore surface & Smooth & Smooth \\
\hline Spore shape & Globose & \\
\hline Spore chain morphology & RA & RA \\
\hline Coagulation of milk & + & + \\
\hline Peptonization of milk & + & + \\
\hline Starch hydrolysis & + & + \\
\hline Melanoid pigment & - & - \\
\hline Nitrate reduction & + & + \\
\hline Lecithinase activity & - & - \\
\hline Gelatin liquefaction & - & - \\
\hline $\mathrm{H}_{2} \mathrm{~S}$ production & - & - \\
\hline $\mathrm{NaCl}$ tolerance & $4 \%$ & \\
\hline \multicolumn{3}{|l|}{ Growth on sole carbon sources $(1.0 \%, w / v)$} \\
\hline Arabinose & + & + \\
\hline $\mathrm{D}(-)$-Fructose & \pm & + \\
\hline $\mathrm{D}(+)$-Xylose & + & + \\
\hline $\mathrm{D}(+)$-Galactose & + & \\
\hline $\mathrm{D}(+)$-Glucose & \pm & + \\
\hline $\mathrm{D}(+)$-Mannose & + & \\
\hline Ribose & + & \\
\hline Sucrose & \pm & + \\
\hline Cellulose & - & \\
\hline Trehalose & \pm & \\
\hline
\end{tabular}

RA, retinaculiaperti; +, positive; -, negative; and \pm , doubtful.

Blank cells: no data available.

It does not utilize cellulose. The organism can tolerate $\mathrm{NaCl}$ up to $4 \%$, exhibit optimum growth at $30^{\circ} \mathrm{C}$, peptonize and coagulate milk, and reduce nitrates to nitrites. It has the ability to hydrolyse starch without ability to produce lecithinase and $\mathrm{H}_{2} \mathrm{~S}$ and to liquefy gelatin (Table 2 ).

The isolate was further identified according to $16 \mathrm{~S}$ rRNA sequencing. The complete $16 \mathrm{~S}$ rRNA gene sequence of actinomycete strain SSHH-1E was determined. Comparisons with other 16S rRNA sequences available in GenBank using BLAST searches were used to select related sequences for constructing a multiple alignment. A phylogenetic tree based on 16S rRNA gene sequences of members of the genus Streptomyces was constructed according to the neighbour-joining method of Saitou and Nei [41] with the software package MEGA4 (Figure 2). The nucleotide sequence of the 16S rRNA gene of the Streptomyces sp. SSHH-1E showed 90\% similarity with other Streptomyces spp. It is clear from Figure 2 that Streptomyces sp. SSHH-1E falls into one distinct subclade with four other species: S. narbonensis (GenBank accession number LN774156.1), S. globisporus (GenBank accession number DQ026634.1), S. roseolus (GenBank accession number NR_041076.1), and S. scabrisporus (GenBank accession 


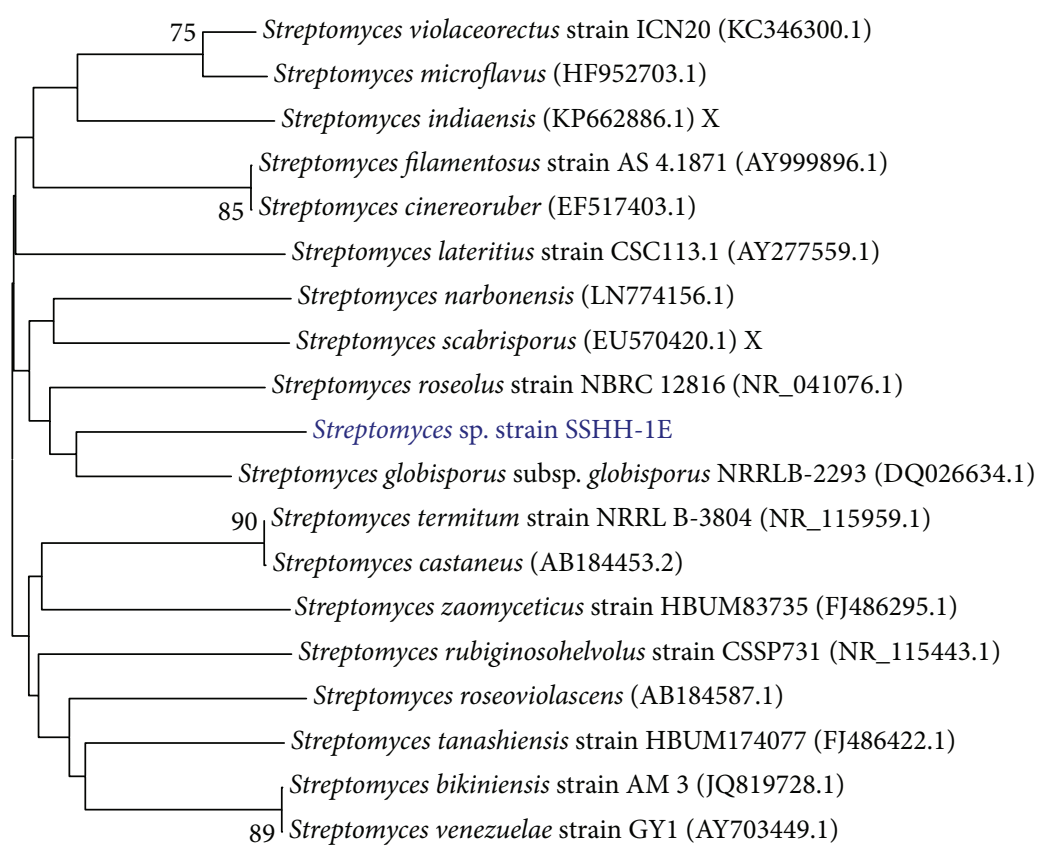

0.5

FIGURE 2: Neighbour-joining tree based on 16S rRNA gene sequences, showing the phylogenetic relationship between Streptomyces sp. SSHH$1 \mathrm{E}$ and 18 related species of the genus Streptomyces. GenBank sequence accession numbers are indicated in parentheses after the strain names. Phylogenetic analysis was conducted in MEGA4.

number EU570420.1). A comparative study between Streptomyces sp. SSHH-1E and its closest phylogenetic neighbours, on the basis of morphological, cultural, and physiological characteristics, was summarized in Table 2. From the taxonomic features, the Streptomyces sp. SSHH-1E was found to closely resemble Streptomyces narbonensis and, thus, it was given the suggested name Streptomyces narbonensis SSHH-1E (GenBank accession number KT160248 (1357 bp)).

3.3. Extracellular Synthesis of AgNPs by Streptomyces narbonensis SSHH-1E. The cell-free filtrate of Streptomyces narbonensis SSHH-1E was pale gray in color, which changed to brown when it was incubated with $1 \mathrm{mM} \mathrm{AgNO}_{3}$ in dark at $30^{\circ} \mathrm{C}$ (Figure 3(a)). The generation of dark brown color is due to the excitation of surface plasmon resonance (SPR) exhibited by the AgNPs [44]. Similar observation was found by ElNaggar et al. [29] for the synthesis of AgNPs by Streptomyces aegyptia NEAE 102 strain by extracellular process.

3.4. UV-Vis Spectrophotometric Analysis. Addition of $\mathrm{AgNO}_{3}$ solution to Streptomyces narbonensis $\mathrm{SSHH}-1 \mathrm{E}$ filtrate led to the appearance of brown color in the solution indicating formation of AgNPs. The UV-visible spectroscopy method was used to quantify this process which has a maximum absorption at $400 \mathrm{~nm}$ (Figure 3(b)); the reaction was completed during the first 24 hours and then it was decreased. The results are similar with that obtained by Motevalli and Zeeb [45] which found the wavelength of the plasma absorption maximum at $400 \mathrm{~nm}$. The results obtained by El-Naggar and Abdelwahed
[46] showed that Streptomyces viridochromogenes has been used for synthesizing AgNPs by extracellular method, and the surface plasmon resonance centered at $400 \mathrm{~nm}$. The synthesis of nanoparticles has not been clearly established by exact mechanism but enzyme NADH-dependent nitrate reductase is known to be involved in the process [47]. The surface plasmon band in the AgNPs solution remains close to $400 \mathrm{~nm}$ throughout the reaction period, suggesting that the particles are dispersed in the aqueous solution with no evidence for aggregation [48]. The results were later confirmed by transmission electron microscopy (TEM).

3.5. Screening of the Significant Variables Affecting AgNPs Biosynthesis Using Plackett-Burman Design. The results of Plackett-Burman design (PBD) (Table 3) indicate that there was a variation of AgNPs production in the twenty trials in the OD range from 0.03 to 1.6 at $400 \mathrm{~nm}$. The relationship between a set of independent variables and the response $(Y)$ is determined by a mathematical model called multiple regression model using Microsoft Excel 2007 to estimate $t$ value, $P$ value, and confidence level. Student's $t$-test for any individual effect allows an evaluation of the probability of finding the observed effect purely by chance. The analysis of regression coefficients and $t$-value of 14 variables were presented in Table 4. With respect to the main effect of each variable (Figure 4), among the 14 variables temperature, $\mathrm{pH}$, inoculum size, inoculum age, medium volume, $\mathrm{NaNO}_{3}$, and yeast extract showed a positive sign of the effect on AgNPs production; however all other factors showed a negative sign 


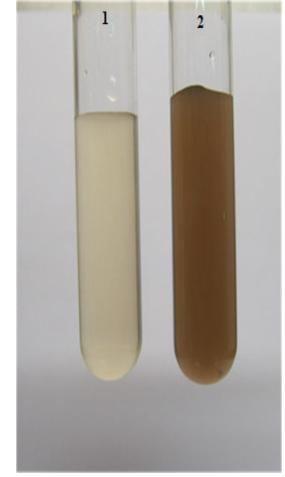

(a)

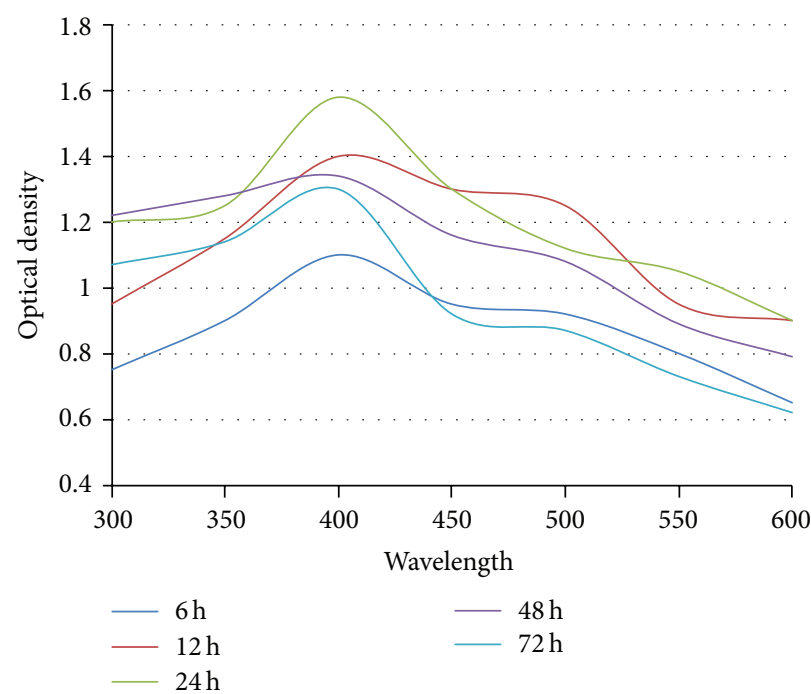

(b)

FIGURE 3: (a) Visible observation of AgNPs biosynthesis by Streptomyces narbonensis SSHH-1E. (1) Cell-free supernatant. (2) After exposure to $\mathrm{AgNO}_{3}$ solution $(1 \mathrm{mM})$. (b) Time course of UV-Vis absorption spectra of AgNPs synthesized by cell-free supernatant of Streptomyces narbonensis SSHH-1E. The absorption spectrum of silver nanoparticles showed a strong broad peak at $400 \mathrm{~nm}$.

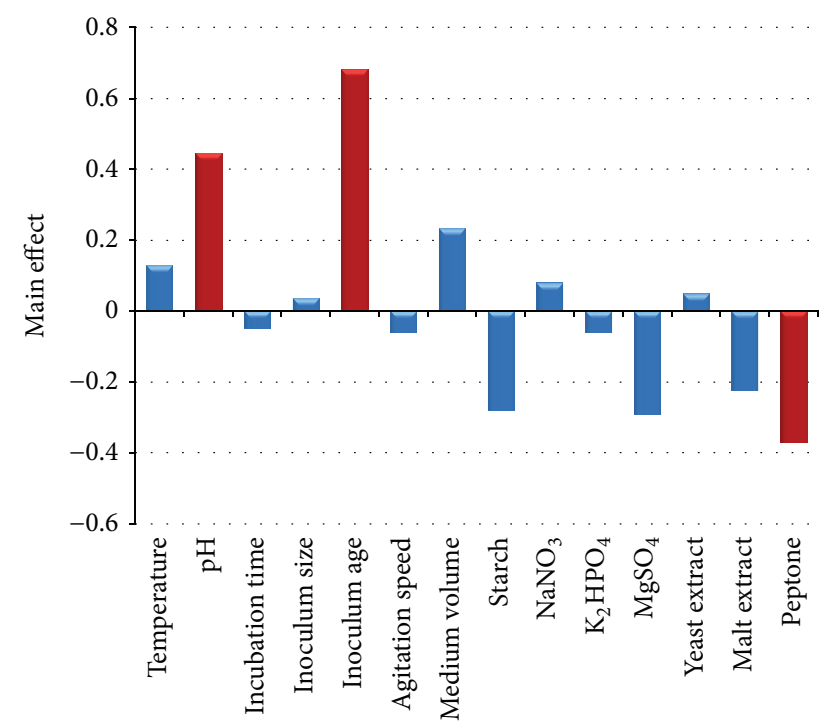

FIgURE 4: The main effects of the process variables on AgNPs biosynthesis by Streptomyces narbonensis SSHH-1E according to the Plackett-Burman experimental results (the red color represents the highly significant variables).

of the effect. When the sign of the effect of the tested variable is positive, the AgNPs production is greater at a high level of the variable, and when the sign is negative, AgNPs production is greater at a low level of the variable [49]. The coefficient of determination, $R^{2}$, was found to be 0.9916 which implied that the sample variation of $99.16 \%$ for AgNPs production was attributed to the independent variables. The coefficient of determination (adjusted $R^{2}$ ) was calculated to be 0.9683 , indicating a good agreement between the experimental and predicted values of AgNPs production. Both of the statistical parameters $t$-value and $P$ value were used to confirm the significance of factors studied. The larger the magnitude of the $t$-value and the smaller the $P$ value, the more significant the corresponding coefficient [50].

The results showed that inoculum age (confidence level, 99.998\%), pH (confidence level, 99.985\%), and peptone (confidence level, 99.666\%) were found as the most important significant factors influencing the AgNPs biosynthesis. On the basis of the confidence level, inoculum age, peptone, and $\mathrm{pH}$ were chosen for further optimization using Box-Behnken statistical design. They were found to exhibit significant effect on AgNPs biosynthesis at the tested concentration levels. The model $F$ value of 42.508 (Table 4 ) implies that the model is significant. The values of significance $P<0.05$ (0.000305) indicate model terms are significant.

By neglecting the terms that were insignificant $(P>0.05)$, the first-order polynomial equation was derived representing AgNPs biosynthesis as a function of the independent variables:

$$
\begin{aligned}
Y_{(\text {AgNPs biosynthesis })=} & 0.485+0.064 X_{1}+0.222 X_{2} \\
& +0.340 X_{5}+0.116 X_{7}-0.140 X_{8} \\
& -0.145 X_{11}-0.109 X_{13} \\
& -0.186 X_{14},
\end{aligned}
$$

where $Y$ is the response and $X_{1}, X_{2}, X_{5}, X_{7}, X_{8}, X_{11}, X_{13}$, and $X_{14}$ are temperature, $\mathrm{pH}$, inoculum age, medium volume, starch, $\mathrm{MgSO}_{4} \cdot 7 \mathrm{H}_{2} \mathrm{O}$, malt extract, and peptone, respectively.

In a confirmatory experiment, to evaluate the accuracy of Plackett-Burman a medium of the composition of $(\mathrm{g} / \mathrm{L})$ 10 starch, $3 \mathrm{NaNO}_{3}, 0.5 \mathrm{~K}_{2} \mathrm{HPO}_{4}, 0.25 \mathrm{MgSO}_{4} \cdot 7 \mathrm{H}_{2} \mathrm{O}, 1$ yeast extract, 1 malt extract, 0.5 peptone adjusting $\mathrm{pH}$ at 8 at $37^{\circ} \mathrm{C}$, medium volume $100 \mathrm{~mL}$, agitation speed $150 \mathrm{rpm}$, inoculum 


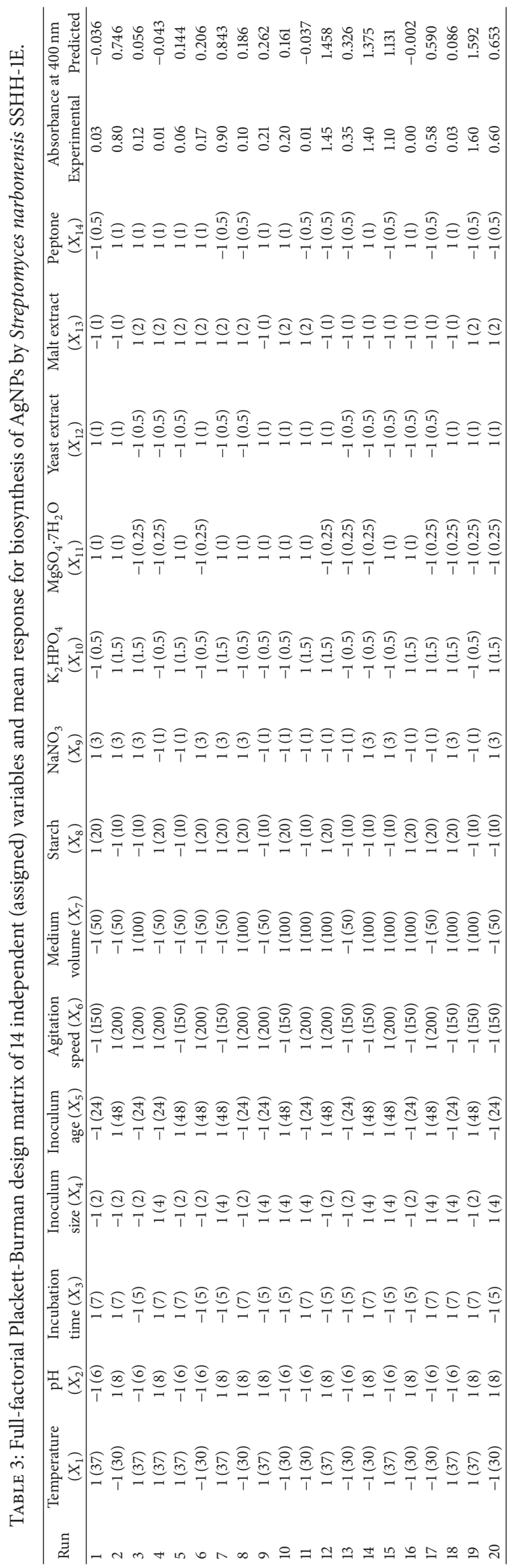


TABLE 4: Estimated regression coefficients for optimization of AgNPs biosynthesis using Plackett-Burman design.

\begin{tabular}{|c|c|c|c|c|c|}
\hline Variables & Coefficients & Main effect & $t$-stat & $P$ value & Confidence level (\%) \\
\hline Intercept & 0.485 & 0.97 & 22.59 & 0.00 & 100.000 \\
\hline Temperature & 0.064 & 0.13 & 3.00 & 0.03 & 96.995 \\
\hline $\mathrm{pH}$ & 0.222 & 0.44 & 10.35 & 0.00 & 99.985 \\
\hline Incubation time & -0.025 & -0.05 & -1.15 & 0.30 & 69.683 \\
\hline Inoculum size & 0.017 & 0.03 & 0.80 & 0.46 & 54.235 \\
\hline Inoculum age & 0.340 & 0.68 & 15.83 & 0.00 & 99.998 \\
\hline Agitation speed & -0.029 & -0.06 & -1.37 & 0.23 & 76.990 \\
\hline Medium volume & 0.116 & 0.23 & 5.39 & 0.00 & 99.703 \\
\hline Starch & -0.140 & -0.28 & -6.52 & 0.00 & 99.873 \\
\hline $\mathrm{NaNO}_{3}$ & 0.040 & 0.08 & 1.85 & 0.12 & 87.656 \\
\hline $\mathrm{K}_{2} \mathrm{HPO}_{4}$ & -0.031 & -0.06 & -1.45 & 0.21 & 79.328 \\
\hline $\mathrm{MgSO}_{4} \cdot 7 \mathrm{H}_{2} \mathrm{O}$ & -0.145 & -0.29 & -6.76 & 0.00 & 99.892 \\
\hline Yeast extract & 0.024 & 0.05 & 1.13 & 0.31 & 69.046 \\
\hline Malt extract & -0.109 & -0.22 & -5.07 & 0.00 & 99.615 \\
\hline Peptone & -0.186 & -0.95 & -8.65 & 0.00 & 99.966 \\
\hline \multicolumn{6}{|c|}{ Analysis of variance (ANOVA) } \\
\hline & df & SS & MS & $F$-test & Significance $F$ ( $P$ value) \\
\hline Regression & 14 & 5.485 & 0.3918 & 42.508 & 0.000305 \\
\hline Residual & 5 & 0.0460 & 0.0092 & & \\
\hline Total & 19 & 5.5312 & & & \\
\hline
\end{tabular}

$t$ : Student's test; $P$ : corresponding level of significance; df: degree of freedom; SS: sum of squares; MS: mean sum of squares; $F$ : Fisher's function; and significance $F$ : corresponding level of significance. Multiple R 0.9958. R square 0.9916. Adjusted R square 0.9683 .

TABLE 5: Full-factorial Box-Behnken design matrix of 3 process variables used in the Box-Behnken design $(K=3)$ with actual factor levels corresponding to coded factor levels and mean response for biosynthesis of AgNPs by Streptomyces narbonensis SSHH-1E.

\begin{tabular}{|c|c|c|c|c|c|}
\hline \multirow{2}{*}{ Run } & \multirow{2}{*}{ Inoculum age $\left(X_{1}\right)$} & \multirow{2}{*}{$\mathrm{pH}\left(X_{2}\right)$} & \multirow{2}{*}{ Peptone $\left(X_{3}\right)$} & \multicolumn{2}{|c|}{ Absorbance at $400 \mathrm{~nm}$} \\
\hline & & & & Experimental & Predicted \\
\hline 1 & $0(48)$ & $1(10)$ & $1(1.5)$ & 4.2 & 4.45 \\
\hline 2 & $1(72)$ & $-1(6)$ & $0(1.0)$ & 1.5 & 1.39 \\
\hline 3 & $1(72)$ & $0(8)$ & $1(1.5)$ & 2.04 & 2.23 \\
\hline 4 & $-1(24)$ & $-1(6)$ & $0(1.0)$ & 2.9 & 3.06 \\
\hline 5 & $-1(24)$ & $0(8)$ & $1(1.5)$ & 4.8 & 4.72 \\
\hline 6 & $0(48)$ & $0(8)$ & $0(1.0)$ & 6.9 & 6.98 \\
\hline 7 & $0(48)$ & $-1(6)$ & $-1(0.5)$ & 3.8 & 3.36 \\
\hline 8 & $0(48)$ & $-1(6)$ & $1(1.5)$ & 1.9 & 1.54 \\
\hline 9 & $1(72)$ & $0(8)$ & $-1(0.5)$ & 3.21 & 3.48 \\
\hline 10 & $0(48)$ & $0(8)$ & $0(1.0)$ & 7.0 & 6.98 \\
\hline 11 & $-1(24)$ & $1(10)$ & $0(1.0)$ & 4.94 & 4.86 \\
\hline 12 & $1(72)$ & $1(10)$ & $0(1.0)$ & 3.24 & 2.90 \\
\hline 13 & $0(48)$ & $0(8)$ & $0(1.0)$ & 7.2 & 6.98 \\
\hline 14 & $0(48)$ & $1(10)$ & $-1(0.5)$ & 3.6 & 3.77 \\
\hline 15 & $0(48)$ & $-1(6)$ & $0(1.0)$ & 3.2 & 3.95 \\
\hline
\end{tabular}

age $48 \mathrm{hr}$, inoculum size $2 \mathrm{v} / \mathrm{v}$, and incubation time 7 days gives OD (AgNPs biosynthesis) 1.6.

3.6. Box-Behnken Design. The results obtained from the 15 experimental runs carried out according to the Box-Behnken design are summarized in Table 5. In this study, a total of 15 experiments with different combination of inoculum age $\left(X_{1}\right), \mathrm{pH}\left(X_{2}\right)$, and peptone $\left(X_{3}\right)$ were performed and the results of experiments for studying the effects of three independent variables on AgNPs biosynthesis are presented. The maximum AgNPs biosynthesis (7.2 OD) was achieved in run number 13, while the minimum AgNPs biosynthesis (1.5 OD) was observed in run number 2 .

3.7. Statistical Analysis, ANOVA, and Model Fitting. The predicted OD of AgNPs production is given in Table 5. The 
TABLE 6: Estimated regression coefficients for optimization of AgNPs biosynthesis using Box-Behnken design.

\begin{tabular}{|c|c|c|c|c|c|}
\hline \multicolumn{2}{|c|}{ Run } & Coefficients & Standard error & $t$-stat & $P$ value \\
\hline \multicolumn{2}{|c|}{ Intercept } & 6.976 & 0.291 & 23.994 & 0.000 \\
\hline \multicolumn{2}{|c|}{ Inoculum age $\left(X_{1}\right)$} & -0.908 & 0.223 & -4.072 & 0.010 \\
\hline \multicolumn{2}{|c|}{$\mathrm{pH}\left(X_{2}\right)$} & 0.828 & 0.175 & 4.725 & 0.005 \\
\hline \multicolumn{2}{|c|}{ Peptone $\left(X_{3}\right)$} & -0.285 & 0.223 & -1.280 & 0.257 \\
\hline \multicolumn{2}{|c|}{$X_{1} X_{2}$} & -0.075 & 0.259 & -0.289 & 0.784 \\
\hline \multicolumn{2}{|c|}{$X_{1} X_{3}$} & -0.339 & 0.363 & -0.935 & 0.393 \\
\hline \multicolumn{2}{|c|}{$X_{2} X_{3}$} & 0.625 & 0.259 & 2.410 & 0.061 \\
\hline \multicolumn{2}{|c|}{$X_{1}^{2}$} & -1.723 & 0.286 & -6.031 & 0.002 \\
\hline \multicolumn{2}{|c|}{$X_{2}^{2}$} & -2.201 & 0.291 & -7.570 & 0.001 \\
\hline \multicolumn{2}{|c|}{$X_{3}^{2}$} & -1.493 & 0.286 & -5.226 & 0.003 \\
\hline \multicolumn{6}{|c|}{ Analysis of variance (ANOVA) } \\
\hline & $\mathrm{df}$ & SS & MS & $F$-test & Significance $F$ ( $P$ value $)$ \\
\hline Regression & 10 & 46.2942 & 4.629 & 14.615 & 0.009911 \\
\hline Residual & 4 & 1.267 & 0.316 & & \\
\hline Total & 14 & 47.5612 & & & \\
\hline
\end{tabular}

$t$ : Student's test; $P$ : corresponding level of significance; df: degree of freedom; SS: sum of squares; MS: mean sum of squares; F: Fisher's function; and significance $F$ : corresponding level of significance. Multiple $R$ 0.9865. $R$ square 0.9733 . Adjusted $R$ square 0.9067 .

coefficient of determination $\left(R^{2}\right)$ of the model was 0.9733 (Table 6), which indicated that the model adequately represented the real relationship between the variables under consideration. $R^{2}$ value of 0.9733 means that $97.33 \%$ of the variability was explained by the model and only $2.77 \%$ of the total variance could not be explained by the model. Therefore, the present $R^{2}$ value reflected a very good fit between the observed and predicted responses and implied that the model is reliable for AgNPs biosynthesis in the present study.

All values of model coefficients were calculated by multiple regression analysis. The significance of each coefficient was determined by Student's $t$-test and $P$ values as listed in Table 6. The $P$ values were used as a tool to check the significance of each of the coefficients which, in turn, are necessary to understand the pattern of the mutual interactions between the test variables. Interpretation of the data was based on the signs (positive or negative effect on the response) and statistical significance of coefficients $(P<0.05)$. Interactions between 2 factors could appear as an antagonistic effect (negative coefficient) or a synergistic effect (positive coefficient). It can be seen from the degree of significance that the linear coefficients of $X_{1}, X_{2}$ and quadratic effect of $X_{1}, X_{2}$, and $X_{3}$ are significant. These values suggest that the inoculum age $\left(X_{1}\right)$ and $\mathrm{pH}\left(X_{2}\right)$ have a direct relationship on the AgNPs biosynthesis. The linear coefficients of $X_{3}$, interaction between $X_{1}$ and $X_{2}$ and $X_{1}$ and $X_{3}$, and interaction between $X_{2}$ and $X_{3}$ are not significant $(P$ value $>0.05)$. The results of the second-order response surface model fitting in the form of analysis of variance (ANOVA) are given in Table 6. ANOVA is required to test the significance and adequacy of the model. The analysis of variance (ANOVA) of the regression model demonstrates that the model is highly significant, as is evident from Fisher's F-test (14.615) and a very low probability value $(0.009911)$.
In order to evaluate the relationship between dependent and independent variables and to determine the maximum AgNPs biosynthesis corresponding to the optimum levels of inoculum age $\left(X_{1}\right), \mathrm{pH}\left(X_{2}\right)$, and peptone $\left(X_{3}\right)$, a secondorder polynomial model was proposed to calculate the optimum levels of these variables. By applying the multiple regression analysis to experimental data, the second-order polynomial equation that defines predicted response $(Y)$ in terms of the independent variables $\left(X_{1}, X_{2}\right.$, and $\left.X_{3}\right)$ was obtained:

$$
\begin{aligned}
Y_{(\mathrm{AgNPs} \text { biosynthesis })}= & 6.976-0.908 X_{1}+0.828 X_{2} \\
& -0.285 X_{3}-0.075 X_{1} X_{2} \\
& -0.339 X_{1} X_{3}+0.625 X_{2} X_{3} \\
& -1.723\left(X_{1}\right)^{2}-2.201\left(X_{2}\right)^{2} \\
& -1.493\left(X_{3}\right)^{2},
\end{aligned}
$$

where $Y$ is the predicted response, $X_{1}$ is the coded value of inoculum age, $X_{2}$ is the coded value of $\mathrm{pH}$, and $X_{3}$ is the coded value of peptone.

The three-dimensional response surface curves which are showed in Figures 5(a)-5(c) were plotted to understand the interaction of the variables and the optimal levels of each variable required for the optimal production. Each figure presents the effect of two factors on AgNPs production, while the third factor was held at constant level. Figure 5(a) illustrates the effects and interaction between $\mathrm{pH}$ value and inoculum age at different concentrations on AgNPs biosynthesis while keeping fixed concentration of peptone at optimum value. The three-dimensional response surface plot indicates that AgNPs biosynthesis was found to increase with 


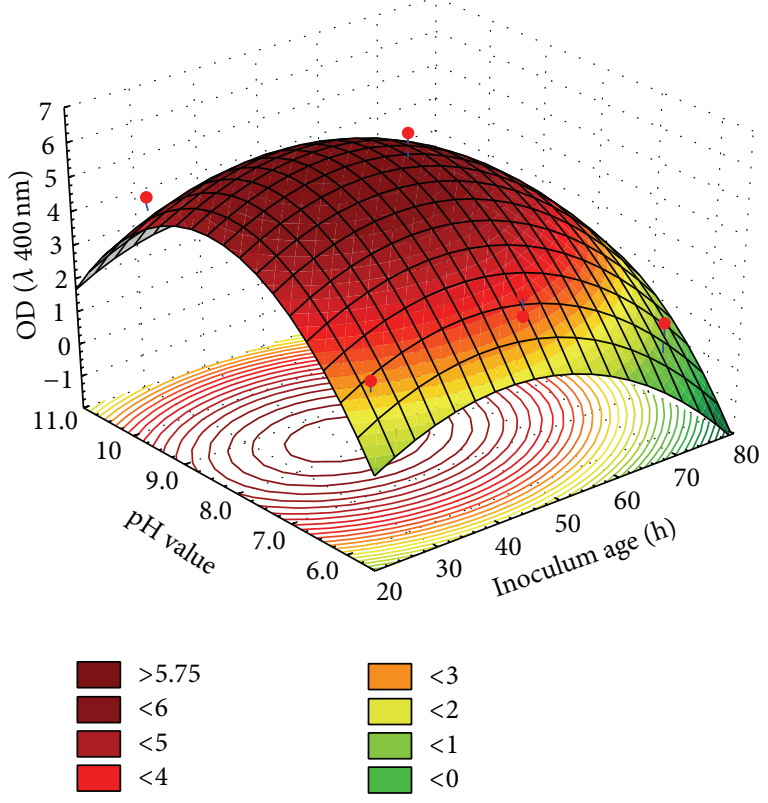

(a)

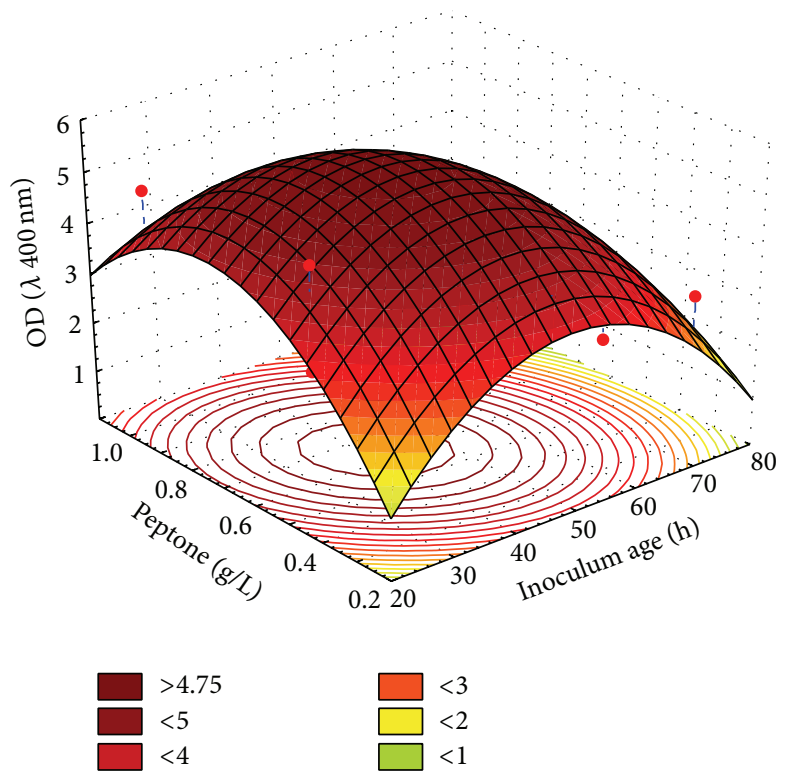

(b)

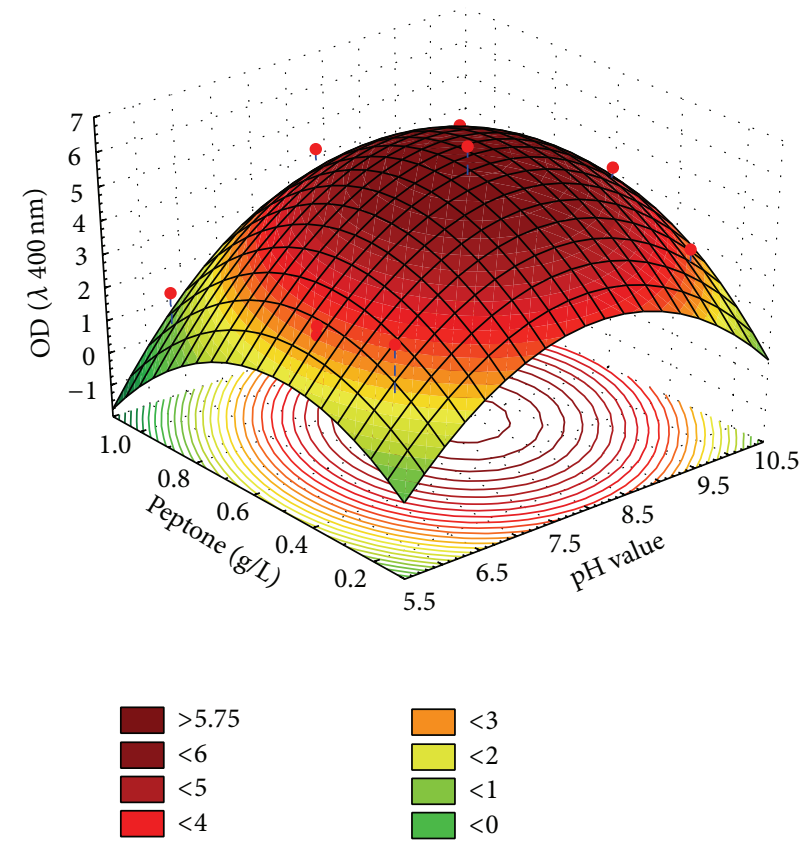

(c)

FIGURE 5: Response surface plots showing the effects of $\mathrm{pH}$, inoculum age, and peptone on AgNPs biosynthesis.

gradual increasing in concentrations of inoculum age and $\mathrm{pH}$ value. The maximum AgNPs biosynthesis was obtained when the middle levels of both inoculum age and $\mathrm{pH}$ value were used. However, further increase in both inoculum age and $\mathrm{pH}$ value resulted in the gradual decrease in the AgNPs biosynthesis. Figure 5(b) represents the effects of peptone concentration and inoculum age on AgNPs biosynthesis when $\mathrm{pH}$ value is fixed at optimum value. It showed that lower and higher levels of the peptone and inoculum age support relatively low levels of AgNPs biosynthesis. On the other hand, the maximum AgNPs biosynthesis is clearly situated close to the central point of the peptone concentration and inoculum age. Figure 5(c) represents AgNPs biosynthesis as a function of $\mathrm{pH}$ and peptone concentration by keeping inoculum age at optimum value. It was observed that there is gradual increasing of the AgNPs biosynthesis with increasing both $\mathrm{pH}$ and peptone concentration. On the other hand, the maximum AgNPs biosynthesis is clearly situated at a middle 
level of both $\mathrm{pH}$ and peptone concentration. Further increase in both $\mathrm{pH}$ and peptone concentration led to the gradual decrease in the AgNPs biosynthesis.

Conventionally, peptone is used in various culture media as major nitrogen source for the organisms. As peptone is partial digestion of protein complex, its carbon atom is liable to be used by organisms. Hence, actinomycetes can use peptone as carbon source when the culture medium lacks carbohydrates (e.g., sugar/s). Voelker and Altaba [51] assayed the role of various nitrogen sources (organic and inorganic) for growth and secondary metabolite production from streptomycetes. During balanced growth, either mineral or organic nitrogen sources were readily utilized without sugar; $0.2-0.5 \%$ peptone concentration is optimum for growth of actinomycetes. Higher peptone concentration disturbs $\mathrm{C} / \mathrm{N}$ ratio and hence retards growth. With glucose, up to $0.8 \%$ of peptone can be used for growth, because glucose, being an alternate to peptone carbon, increases the $\mathrm{C} / \mathrm{N}$ ratio to the safe level. The $\mathrm{pH}$ of the cultivation medium is very important for the growth of microorganisms and characteristic of their metabolism and, hence, for the biosynthesis of metabolites. The hydrogen ion concentration may have a direct effect on the cell, or it may indirectly affect it by varying the dissociation degree of the medium components [52]. Solingen et al. [53] reported an alkaline novel Streptomyces species isolated from east African soda lakes that have an optimal $\mathrm{pH} 8$, highlighting the effect of alkaline environment on the adaptation of Streptomyces species. The higher inoculum density is inhibitory to the biproduct synthesis as too much biomass can deplete the substrate nutrients or accumulation of some nonvolatile self-inhibiting substances that inhibit the product formation [54] and lower density may give insufficient biomass causing induced product formation whereas higher inoculum may produce too much biomass which is inhibitory to the product formation [55].

3.8. Verification of the Model. The validity of the results predicted by the regression model was confirmed by carrying out repeated experiments under optimal fermentation conditions (i.e., inoculum age $(48 \mathrm{~h})$, peptone $(1.0 \mathrm{gm})$, and $\mathrm{pH}(8))$. The results obtained from three replications demonstrated that the average of the maximum OD $(7.2$ at $400 \mathrm{~nm})$ obtained was close to the predicted OD (6.98). The verification revealed a high degree of accuracy of the model (more than 96.9\%), indicating the model validation under the tested conditions. The excellent correlation between the predicted and measured values from these experiments indicates validity of response model.

\subsection{Characterization of AgNPs Synthesized by Streptomyces narbonensis SSHH-1E}

3.9.1. Transmission Electron Microscopy (TEM). Transmission electron microscopy is a powerful method to determine the morphology and size of nanostructures. Transmission electron microscopy micrographs of the synthesized AgNPs revealed the formation of extracellularly spherical nanoparticles with a size range of $20-40 \mathrm{~nm}$ (Figure 6). The results

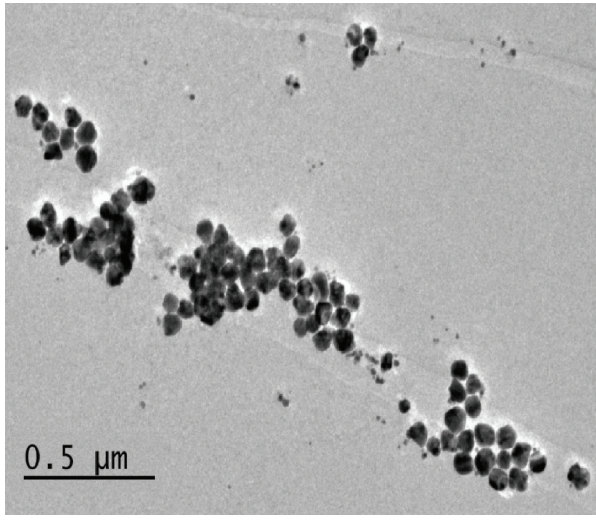

FIgURE 6: TEM image of the AgNPs formed by the reaction of $1 \mathrm{mM}$ $\mathrm{AgNO}_{3}$ and the cell-free culture supernatant of Streptomyces narbonensis SSHH-1E. Scale bar $=0.5 \mu \mathrm{m}$.

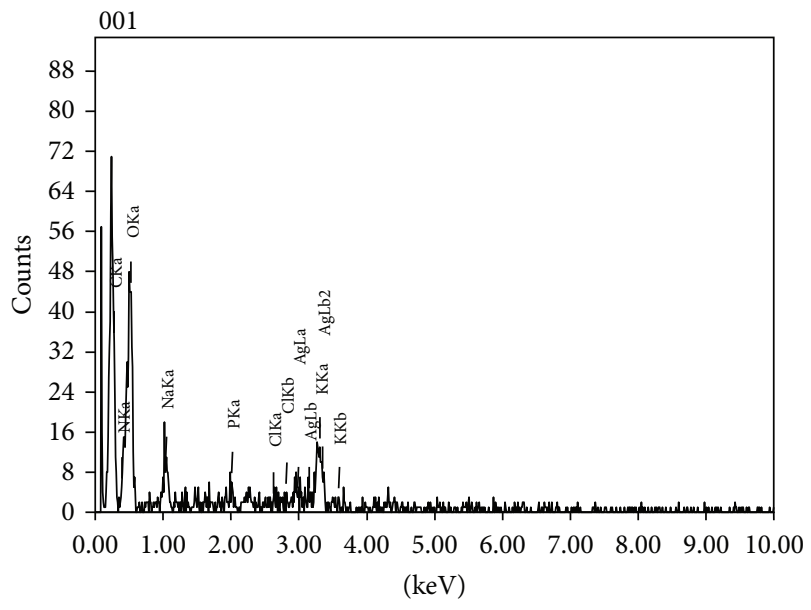

FIGURE 7: EDX spectrum showing a peak between 3 and $4 \mathrm{keV}$, confirming the presence of silver.

obtained by Raut et al. [56] revealed that the AgNPs are predominantly spherical in shape.

\subsubsection{Energy Dispersive X-ray (EDX) Spectroscopy. EDX} analysis confirmed silver as the major constituent element (Figure 7). The spectrum at $3 \mathrm{keV}$ indicates a strong signal for silver which is characteristic of nanosized metallic silver [57]. In addition, other peaks for N, C, and O were observed which are possibly due to emissions from proteins or enzymes present in culture-free supernatant [58]. Peaks for $\mathrm{P}, \mathrm{K}$, and $\mathrm{Cl}$ were also observed.

\subsubsection{Fourier Transformed Infrared (FTIR) Spectroscopy} Analysis. Fourier transformed infrared spectroscopy is useful in probing the chemical composition of the surface of the AgNPs and the local molecular environment of the capping agents on the nanoparticles [59] or identifying the possible biomolecules responsible for the reduction of the $\mathrm{Ag}^{+}$ions by the cell filtrate [60]. The FTIR spectrum of AgNPs (Figure 8) manifests seven absorption peaks. The presence of bands 


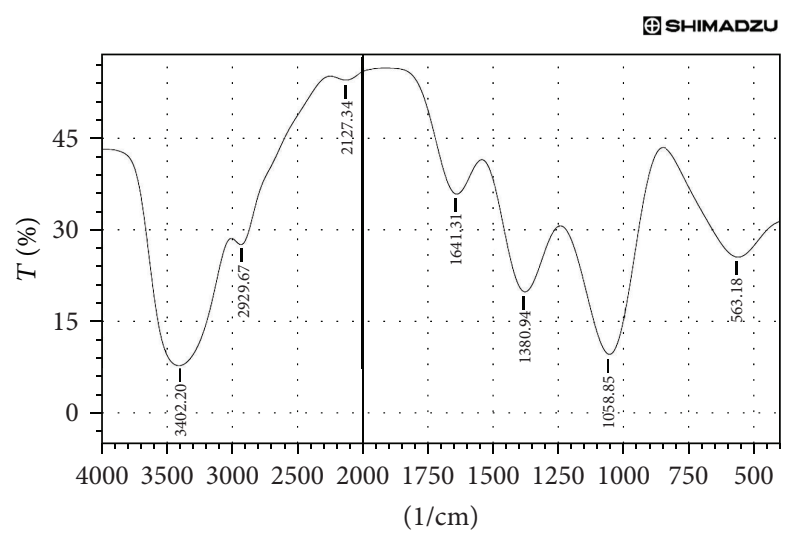

FIGURE 8: FTIR spectrum recorded from a drop-coated film of AgNPs synthesized by Streptomyces narbonensis SSHH-1E.

at $1058.85 \mathrm{~cm}^{-1}$ in the FTIR spectrum suggests the capping agent of biosynthesized nanoparticles possesses an aromatic amine group by El-Naggar et al. [29]. The band at $3402.2 \mathrm{~cm}^{-1}$ shows $\mathrm{O}-\mathrm{H}$ stretching vibrations of hydroxyl groups [61]. The band at $1380.94 \mathrm{~cm}^{-1}$ assigned to $\mathrm{O}-\mathrm{H}$ bend indicates carboxylate [61]. The peak at $2127.34 \mathrm{~cm}^{-1}$ shows C-N stretching for amines; the peak at $1641.31 \mathrm{~cm}^{-1}$ indicates the presence of carbonyl group [62]. A weak band at $2929.67 \mathrm{~cm}^{-1}$ corresponds to $\mathrm{C}-\mathrm{H}$ stretch alkanes [63] and $\mathrm{O}-\mathrm{H}$ stretch carboxylic acids [64]. The peaks at range $561-568 \mathrm{~cm}^{-1}$ represent the vibration peaks of $\mathrm{PO}_{4}{ }^{3-}$ by Maisara et al. [65]. The FTIR spectra, which give rise to the well-known signatures in the infrared region of the electromagnetic spectrum according to the presence of different functional groups like $\mathrm{C}-\mathrm{N}, \mathrm{C}-\mathrm{O}-\mathrm{C}$, amide linkages, and -COO-, may be between amino acid residues in protein and synthesized AgNPs. Our findings were confirmed by Gole et al. [66] who reported stabilization of the AgNPs by proteins which can bind to nanoparticles through either free amine groups or residues in the proteins and through the electrostatic attraction of negatively charged carboxylate groups in enzymes present in the cell-free supernatant. Sastry et al. [67] have reported that bonds or functional groups such as -C-O-C-, -C-O-, and -C=C- are derived from heterocyclic compounds like proteins, which are present as the capping ligands of the nanoparticles [68].

3.10. Antimicrobial Activity of AgNPs Suspension Loaded onto Cotton Fabrics by Disc Diffusion Method. The fabricated cotton cloth which is loaded with AgNPs was tested for their antimicrobial activity against $S$. aureus, E. coli, and C. albicans using agar well diffusion method. After $24 \mathrm{hr}$ incubation time, a zone of inhibition was observed against the tested organism (Table 7) showing inhibition zone of $16 \mathrm{~mm}$ against $S$. aureus, while against $E$. coli it was recorded as $18 \mathrm{~mm}$; finally the inhibition zone diameter against C. albicans was $13 \mathrm{~mm}$ (Figure 9). The presence of zone of inhibition indicates that the antimicrobial is leaching into the agar. The mechanism of the inhibitory effects of $\mathrm{Ag}^{+}$ions on microorganisms is partially known. It is reported that the positively charged ionic form of the silver is highly toxic for microorganisms
TABLE 7: Antimicrobial activity of AgNPs produced by Streptomyces narbonensis SSHH-1E loaded on cotton fabrics.

\begin{tabular}{lc}
\hline Microorganisms & Inhibition zone $(\mathrm{mm})$ \\
\hline E. coli (Gram-negative) & 18 \\
S. aureus (Gram-positive) & 16 \\
C. albicans (yeast) & 13 \\
\hline
\end{tabular}

as it can attract the negatively charged cell membrane of microorganisms through the electrostatic interaction [69, 70]. Due to their unique size and greater surface area, silver nanoparticles can easily reach the nuclear content of bacteria $[71,72]$. A survey of the literature showed that the electrostatic attraction between negatively charged bacterial cells and positively charged nanoparticles was crucial for the antibacterial activity [73]. These results are very close to that obtained by Sourav Ghosh et al. [74]. The fabricated cotton cloth which is loaded with AgNPs was tested for their antimicrobial activity at different time intervals. Produced AgNPs showed antimicrobial efficacy for a long time after its synthesis.

3.11. Stability and Dispersibility of Synthesized Silver Nanoparticles. The stability of the biosynthesized AgNPs was observed optically by a color change. There was no significant change in color or appearance of agglomeration over a period of more than 6 months, which indicates high stability of the biosynthesized AgNPs. Such observations were also checked using UV-visible spectroscopy and transmission electron microscopy. The stability and the AgNPs dispersibility with increasing of the storage time were checked by transmission electron microscopy, whose results are in good agreement with UV-visible spectroscopy studies. No considerable changes in size, distribution, and morphology between the fresh and stored samples were observed. Therefore, we can conclude that the Ag nanoparticles are stable. FTIR spectrum supports the presence of protein compounds on the surface of biosynthesized nanoparticles. It has been suggested that stability of the AgNPs generated using cell-free culture supernatants could be due to the presence of a proteinaceous capping agent that can bind to nanoparticles and prevents aggregation of the silver nanoparticles [63].

\section{Conclusion}

It can be concluded that Streptomyces narbonensis SSHH$1 \mathrm{E}$ is an excellent microbial resource for the synthesis of AgNPs. The cotton fabrics incorporating AgNPs were an efficient method for the preparation of antimicrobial fabrics. These fabrics show biocidal action against E. coli, S. aureus, and $C$. albicans, thus showing great potential to be used as antiseptic dressing or bandage, which are in high demand for biomedical applications. The biosynthesized AgNPs were characterized by transmission electron microscopy, dispersive X-ray analysis, and Fourier transformed infrared spectroscopy analysis. Statistical optimization of fermentation conditions using Plackett-Burman design and Box-Behnken design appears to be a valuable tool for the production of AgNPs by Streptomyces narbonensis SSHH-1E. A 4.5-fold 


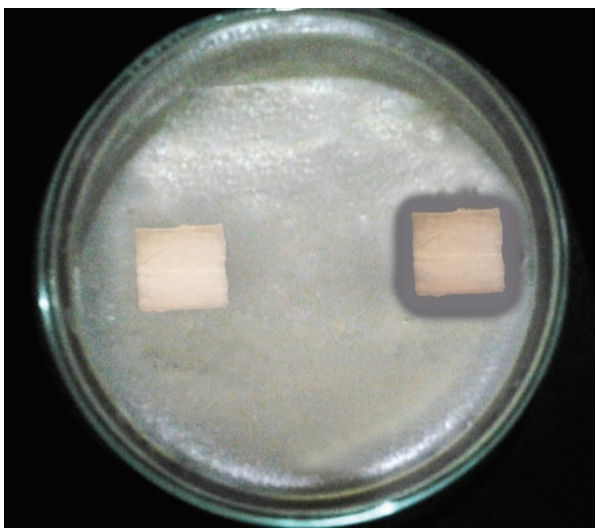

E. coli

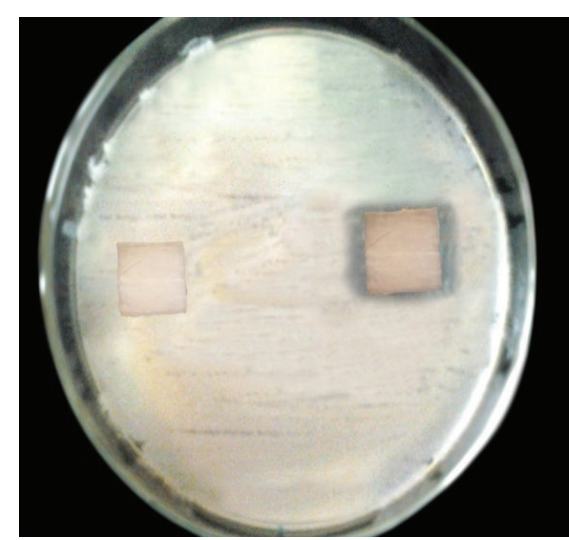

Staphylococcus aureus

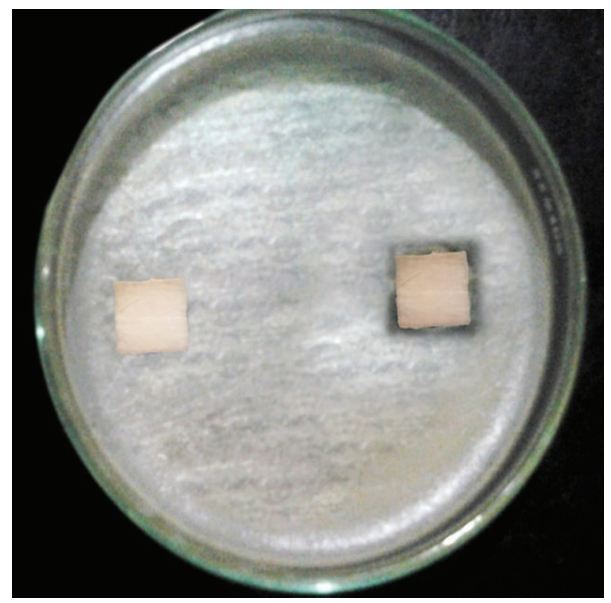

Candida albicans

Figure 9: Antimicrobial activities of cotton fabrics loaded with AgNPs.

increase in AgNPs production was achieved with the following optimized factors: inoculums age $(48 \mathrm{~h})$, peptone $(0.5 \mathrm{~g} /$ $\mathrm{L})$, and $\mathrm{pH}$ value (8).

\section{Competing Interests}

The authors declare that there is no conflict of interests regarding the publication of this paper.

\section{References}

[1] M. Valodkar, A. Bhadoria, J. Pohnerkar, M. Mohan, and S. Thakore, "Morphology and antibacterial activity of carbohydrate-stabilized silver nanoparticles," Carbohydrate Research, vol. 345, no. 12, pp. 1767-1773, 2010.

[2] D. S. Balaji, S. Basavaraja, R. Deshpande, D. B. Mahesh, B. K. Prabhakar, and A. Venkataraman, "Extracellular biosynthesis of functionalized silver nanoparticles by strains of Cladosporium cladosporioides fungus," Colloids and Surfaces B: Biointerfaces, vol. 68, no. 1, pp. 88-92, 2009.

[3] K. Kalishwaralal, V. Deepak, S. R. K. Pandian et al., "Biosynthesis of silver and gold nanoparticles using Brevibacterium casei," Colloids and Surfaces B: Biointerfaces, vol. 77, no. 2, pp. 257-262, 2010.
[4] M. V. Roldán, A. L. Frattini, O. A. Sanctis, and N. S. Pellegrini, "Synthesis of silver nanoparticles by chemical reduction method," ANALES Asociación Física Argentina, vol. 17, pp. 212217, 2005.

[5] Z. Zhu, L. Kai, and Y. Wang, "Synthesis and applications of hyperbranched polyesters-preparation and characterization of crystalline silver nanoparticles," Materials Chemistry and Physics, vol. 96, no. 2-3, pp. 447-453, 2006.

[6] A. S. Edelstein and R. C. Cammaratra, Eds., Nanomaterials: Synthesis, Properties, and Applications, IOP Publishers, Bristol, UK, 1996.

[7] M. Maillard, S. Giorgio, and M.-P. Pileni, "Silver nanodisks," Advanced Materials, vol. 14, no. 15, pp. 1084-1086, 2002.

[8] N. Durán, P. D. Marcato, O. L. Alves, G. I. H. De Souza, and E. Esposito, "Mechanistic aspects of biosynthesis of silver nanoparticles by several Fusarium oxysporum strains," Journal of Nanobiotechnology, vol. 3, article 8, 7 pages, 2005.

[9] M. Rai, A. Yadav, and A. Gade, "Silver nanoparticles as a new generation of antimicrobials," Biotechnology Advances, vol. 27, no. 1, pp. 76-83, 2009.

[10] N. Akaighe, R. I. MacCuspie, D. A. Navarro et al., "Humic acidinduced silver nanoparticle formation under environmentally relevant conditions," Environmental Science and Technology, vol. 45, no. 9, pp. 3895-3901, 2011. 
[11] M. Kowshik, S. Ashtaputre, S. Kharrazi et al., "Extracellular synthesis of silver nanoparticles by a silver-tolerant yeast strain MKY3," Nanotechnology, vol. 14, no. 1, pp. 95-100, 2003.

[12] B. Tomšič, B. Simončič, B. Orel et al., "Antimicrobial activity of $\mathrm{AgCl}$ embedded in a silica matrix on cotton fabric," Carbohydrate Polymers, vol. 75, no. 4, pp. 618-626, 2009.

[13] G. I. H. Souza, P. D. Marcato, N. Durán, and E. Esposito, "Utilization of Fusarium oxysporum in the biosynthesis of silver nanoparticles and its antibacterial activities," in Proceedings of the 9th National Meeting of Environmental Microbiology, Curtiba, Brazil, 2004.

[14] X. Wang, S. Li, H. Yu, and J. Yu, "In situ anion-exchange synthesis and photocatalytic activity of $\mathrm{Ag}_{8} \mathrm{~W}_{4} \mathrm{O}_{16} / \mathrm{AgCl}$-nanoparticle core-shell nanorods," Journal of Molecular Catalysis A: Chemical, vol. 334, no. 1-2, pp. 52-59, 2011.

[15] F. Furno, K. S. Morley, B. Wong et al., "Silver nanoparticles and polymeric medical devices: a new approach to prevention of infection?" Journal of Antimicrobial Chemotherapy, vol. 54, no. 6, pp. 1019-1024, 2004.

[16] S. Schultz, D. R. Smith, J. J. Mock, and D. A. Schultz, "Singletarget molecule detection with nonbleaching multicolor optical immunolabels," Proceedings of the National Academy of Sciences of the United States of America, vol. 97, no. 3, pp. 996-1001, 2000.

[17] M. S. M. Peterson, J. Bouwman, A. Chen, and M. Deutsch, "Inorganic metallodielectric materials fabricated using two single-step methods based on the Tollen's process," Journal of Colloid and Interface Science, vol. 306, no. 1, pp. 41-49, 2007.

[18] X. Sun and Y. Luo, "Preparation and size control of silver nanoparticles by a thermal method," Materials Letters, vol. 59, no. 2930, pp. 3847-3850, 2005.

[19] K. Shao and J.-N. Yao, "Preparation of silver nanoparticles via a non-template method," Materials Letters, vol. 60, no. 29-30, pp. 3826-3829, 2006.

[20] T. Tsuji, K. N. Iryo, N. Watanabe, and M. Tsuji, "Preparation of silver nanoparticles by laser ablation in solution: influence of laser wavelength on particle size," Applied Surface Science, vol. 202, no. 1-2, pp. 80-85, 2002.

[21] D. Bhattacharya and R. K. Gupta, "Nanotechnology and potential of microorganisms," Critical Reviews in Biotechnology, vol. 25, no. 4, pp. 199-204, 2005.

[22] V. C. Verma, R. N. Kharwar, and A. C. Gange, "Biosynthesis of noble metal nanoparticles and their application, CAB Review: perspectives in agriculture, Vatenary science," Nutrition. National Resource, vol. 4, no. 26, pp. 1-17, 2009.

[23] K. B. Narayanan and N. Sakthivel, "Biological synthesis of metal nanoparticles by microbes," Advances in Colloid and Interface Science, vol. 156, no. 1-2, pp. 1-13, 2010.

[24] P. Mohanpuria, N. K. Rana, and S. K. Yadav, "Biosynthesis of nanoparticles: technological concepts and future applications," Journal of Nanoparticle Research, vol. 10, no. 3, pp. 507-517, 2008.

[25] D. Mandal, M. E. Bolander, D. Mukhopadhyay, G. Sarkar, and P. Mukherjee, "The use of microorganisms for the formation of metal nanoparticles and their application," Applied Microbiology and Biotechnology, vol. 69, no. 5, pp. 485-492, 2006.

[26] N. Y. Tsibakhashvili, E. I. Kirkesali, D. T. Pataraya et al., "Microbial synthesis of silver nanoparticles by Streptomyces glaucus and Spirulina platensis," Nanomaterials: Applications and Properties, vol. 2, pp. 306-310, 2011.

[27] R. Das, S. Gang, and S. S. Nath, "Preparation and antibacterial activity of silver nanoparticles," Journal of Biomaterials and Nanobiotechnology, vol. 2, no. 4, pp. 472-475, 2011.
[28] A. Ahmad, P. Mukherjee, S. Senapati et al., "Extracellular biosynthesis of silver nanoparticles using the fungus Fusarium oxysporum," Colloids and Surfaces B: Biointerfaces, vol. 28, no. 4, pp. 313-318, 2003.

[29] N. E.-A. El-Naggar, N. A. M. Abdelwahed, and O. M. M. Darwesh, "Fabrication of biogenic antimicrobial silver nanoparticles by Streptomyces aegyptia NEAE 102 as eco-friendly nanofactory," Journal of Microbiology and Biotechnology, vol. 24, no. 4, pp. 453-464, 2014.

[30] S. A. Masurkar, P. R. Chaudhari, V. B. Shidore, and S. P. Kamble, "Rapid biosynthesis of silver nanoparticles using Cymbopogan citratus (Lemongrass) and its antimicrobial activity," NanoMicro Letters, vol. 3, no. 3, pp. 189-194, 2011.

[31] A. A. Khardenavis, A. Kapley, and H. J. Purohit, "Processing of poultry feathers by alkaline keratin hydrolyzing enzyme from Serratia sp. HPC 1383," Waste Management, vol. 29, no. 4, pp. 1409-1415, 2009.

[32] K. K. Prasad, S. V. Mohan, R. S. Rao, B. R. Pati, and P. N. Sarma, "Laccase production by Pleurotus ostreatus 1804: optimization of submerged culture conditions by Taguchi DOE methodology," Biochemical Engineering Journal, vol. 24, no. 1, pp. 17-26, 2005.

[33] V. V. R. Bandaru, S. R. Somalanka, D. R. Mendu, N. R. Madicherla, and A. Chityala, "Optimization of fermentation conditions for the production of ethanol from sago starch by coimmobilized amyloglucosidase and cells of Zymomonas mobilis using response surface methodology," Enzyme and Microbial Technology, vol. 38, no. 1-2, pp. 209-214, 2006.

[34] R. M. Banik, A. Santhiagu, and S. N. Upadhyay, "Optimization of nutrients for gellan gum production by Sphingomonas paucimobilis ATCC-31461 in molasses based medium using response surface methodology," Bioresource Technology, vol. 98, no. 4, pp. 792-797, 2007.

[35] R. L. Plackett and J. P. Burnam, "The design of optimum multifactorial experiments," Biometrika, vol. 33, pp. 305-325, 1946.

[36] G. E. Box and K. B. Wilson, "On the experimental attainment of optimum conditions," Journal of the Royal Statistical Society B. Methodological, vol. 13, pp. 1-45, 1951.

[37] E. B. Shirling and D. Gottlieb, "Methods for characterization of Streptomyces species," International Journal of Systematic Bacteriology, vol. 16, no. 3, pp. 313-340, 1966.

[38] J. Sambrook, E. F. Fritsch, and T. Maniatis, Molecular Cloning: a Laboratory Manual, Cold Spring Harbor Laboratory, Cold Spring Harbor, NY, USA, 2nd edition, 1989.

[39] S. F. Altschul, T. L. Madden, A. A. Schäffer et al., "Gapped BLAST and PSI-BLAST: a new generation of protein database search programs," Nucleic Acids Research, vol. 25, no. 17, pp. 3389-3402, 1997.

[40] K. Tamura, J. Dudley, M. Nei, and S. Kumar, "MEGA4: molecular evolutionary genetics analysis (MEGA) software version 4.0," Molecular Biology and Evolution, vol. 24, no. 8, pp. 15961599, 2007.

[41] N. Saitou and M. Nei, "The neighbor-joining method: a new method for reconstructing phylogenetic trees," Molecular Biology and Evolution, vol. 4, no. 4, pp. 406-425, 1987.

[42] N. Durán, P. D. Marcato, G. I. H. De Souza, O. L. Alves, and E. Esposito, "Antibacterial effect of silver nanoparticles produced by fungal process on textile fabrics and their effluent treatment," Journal of Biomedical Nanotechnology, vol. 3, no. 2, pp. 203-208, 2007.

[43] S. Abdeen, S. Geo, P. P. K. Sukanya, and R. P. Dhanya, "Biosynthesis of silver nanoparticles from actinomycetes for therapeutic 
applications," International Journal of Nano Dimension, vol. 5, no. 2, pp. 155-162, 2014.

[44] P. Phanjom and G. Ahmed, "Biosynthesis of silver nanoparticles by Aspergillus oryzae (MTCC No. 1846) and its characterizations," Nanoscience and Nanotechnology, vol. 5, no. 1, pp. 14-21, 2015.

[45] K. Motevalli and M. Zeeb, "Dispersive liquid-liquid microextraction using silver nanoparticles as electrostatic probes for preconcentartion and quantitative analysis of terazosin," International Journal of Nano Dimension, vol. 1, no. 3, pp. 187-201, 2011.

[46] N. E.-A. El-Naggar and N. A. M. Abdelwahed, "Application of statistical experimental design for optimization of silver nanoparticles biosynthesis by a nanofactory Streptomyces viridochromogenes," Journal of Microbiology, vol. 52, no. 1, pp. 53-63, 2014.

[47] J. Y. Roh, S. J. Sim, J. Yi, K. Park, K. H. Chung, and D. Y. Ryu, "Ecotoxicity of silver nanoparticles on the soil nematode Caenorhabditis elegans using functional ecotoxicogenomics," Environmental Science \& Technology, vol. 43, no. 10, pp. 39333940, 2009.

[48] M. A. Faramarzi and H. Forootanfar, "Biosynthesis and characterization of gold nanoparticles produced by laccase from Paraconiothyrium variabile," Colloids and Surfaces B: Biointerfaces, vol. 87, no. 1, pp. 23-27, 2011.

[49] D. Gangadharan, S. Sivaramakrishnan, K. M. Nampoothiri, R. K. Sukumaran, and A. Pandey, "Response surface methodology for the optimization of alpha amylase production by Bacillus amyloliquefaciens," Bioresource Technology, vol. 99, no. 11, pp. 4597-4602, 2008.

[50] R. H. Myers and R. C. Montgomery, Response Surface Methodology: Process and Product Optimization Using Designed Experiments, John Wiley \& Son, New York, NY, USA, 2002.

[51] F. Voelker and S. Altaba, "Nitrogen source governs the patterns of growth and pristinamycin production in 'Streptomyces pristinaespiralis"” Microbiology, vol. 147, no. 9, pp. 2447-2459, 2001.

[52] P. Jain and R. K. Pundir, "Effect of fermentation medium, $\mathrm{pH}$ and temperature variations on antibacterial soil fungal metabolite production," Journal of Agricultural Science and Technology, vol. 7, no. 2, pp. 247-269, 2011.

[53] P. V. Solingen, D. Meijer, W. A. Kleij et al., "Cloning and expression of an endocellulase gene from a novel streptomycete isolated from an East African soda lake," Extremophiles, vol. 5, no. 5, pp. 333-341, 2001.

[54] Z. Baysal, F. Uyar, and Ç. Aytekin, "Solid state fermentation for production of $\alpha$-amylase by a thermotolerant Bacillus subtilis from hot-spring water," Process Biochemistry, vol. 38, no. 12, pp. 1665-1668, 2003.

[55] R. E. Mudgett, "Solid state fermentations," in Manual of Industrial Microbiology and Biotechnology, A. L. Demain and N. A. Solomon, Eds., vol. 66, p. 83, American Society for Microbiology, Washington, DC, USA, 1986.

[56] R. W. Raut, J. R. Lakkakula, N. S. Kolekar, V. D. Mendhulkar, and S. B. Kashid, "Phytosynthesis of silver nanoparticle using Gliricidia sepium (Jacq.)," Current Nanoscience, vol. 5, no. 1, pp. 117-122, 2009.

[57] P. Magudapathy, P. Gangopadhyay, B. K. Panigrahi, K. G. M. Nair, and S. Dhara, "Electrical transport studies of Ag nanoclusters embedded in glass matrix," Physica B: Condensed Matter, vol. 299, no. 1-2, pp. 142-146, 2001.

[58] P. Mukherjee, A. Ahmad, D. Mandal et al., "Fungus-mediated synthesis of silver nanoparticles and their immobilization in the mycelial matrix: a novel biological approach to nanoparticle synthesis," Nano Letters, vol. 1, no. 10, pp. 515-519, 2001.

[59] S. A. Umoren, I. B. Obot, and Z. M. Gasem, "Green synthesis and characterization of silver nanoparticles using red apple (Malus domestica) fruit extract at room temperature," Journal of Materials and Environmental Science, vol. 5, no. 3, pp. 907-914, 2014.

[60] N. K. S. Hemath, G. Kumar, L. Karthik, and R. K. V. Bhaskara, "Extracellular biosynthesis of silver nanoparticles using the filamentous fungus Penicillium sp," Archives of Applied Sciece Research, vol. 2, no. 6, pp. 161-167, 2010.

[61] L. Rastogi and J. Arunachalam, "Sunlight based irradiation strategy for rapid green synthesis of highly stable silver nanoparticles using aqueous garlic (Allium sativum) extract and their antibacterial potential," Materials Chemistry and Physics, vol. 129, no. 1-2, pp. 558-563, 2011.

[62] S. Ghosh, S. Patil, M. Ahire et al., "Synthesis of silver nanoparticles using Dioscorea bulbifera tuber extract and evaluation of its synergistic potential in combination with antimicrobial agents," International Journal of Nanomedicine, vol. 7, pp. 483-496, 2012.

[63] A. Mohamedin, N. E.-A. El-Naggar, S. S. Hamza, and A. A. Sherief, "Green synthesis, characterization and antimicrobial activities of silver nanoparticles by Streptomyces viridodiastaticus SSHH-1 as a living nano-factory: statistical optimization of process variables," Current Nanoscience, vol. 11, no. 5, pp. 640654, 2015.

[64] S. Rajeshkumar, C. Kannan, and G. Annadurai, "Synthesis and characterization of antimicrobial silver nanoparticles using marine brown seaweed Padina tetrastromatica," Drug Invention Today, vol. 4, no. 10, pp. 511-513, 2012.

[65] S. M. A. Maisara, M. L. Pat, and K. H. Lee, "Synthesis and characterization of hydroxyapatite nanoparticles and $\beta$-TCP particles," in 2nd International Conferences on Biotechnology and Food Science, vol. 7, pp. 184-188, IACSIT Press, Singapore, 2011.

[66] A. Gole, C. Dash, V. Ramakrishnan et al., "Pepsin-gold colloid conjugates: preparation, characterization, and enzymatic activity," Langmuir, vol. 17, no. 5, pp. 1674-1679, 2001.

[67] M. Sastry, A. Ahmad, M. I. Khan, and R. Kumar, "Biosynthesis of metal nanoparticles using fungi and actinomycete," Current Science, vol. 85, no. 2, pp. 162-170, 2003.

[68] N. Y. Tsibakhashvili, E. I. Kirkesali, D. T. Pataraya et al., "Microbial synthesis of silver nanoparticles by Streptomyces glaucus and Spirulina platensis," Advanced Science Letters, vol. 4, no. 1112, pp. 3408-3417, 2011.

[69] P. Dibrov, J. Dzioba, K. K. Gosink, and C. C. Häse, "Chemiosmotic mechanism of antimicrobial activity of $\mathrm{Ag}^{+}$in Vibrio cholerae," Antimicrobial Agents and Chemotherapy, vol. 46, no. 8, pp. 2668-2670, 2002.

[70] T. Hamouda, A. Myc, B. Donovan, A. Y. Shih, J. D. Reuter, and J. R. Baker Jr., "A novel surfactant nanoemulsion with a unique non-irritant topical antimicrobial activity against bacteria, enveloped viruses and fungi," Microbiological Research, vol. 156, no. 1, pp. 1-7, 2001.

[71] S. F. Chen, J. P. Li, K. Quin et al., "Large scale photochemical

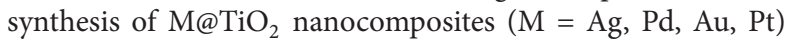
and their optical properties, $\mathrm{CO}$ oxidation performance, and antibacterial effect," Nano Research, vol. 3, no. 4, pp. 244-255, 2010.

[72] B. Chudasama, A. K. Vala, N. Andhariya, R. V. Upadhyay, and R. V. Mehta, "Enhanced antibacterial activity of bifunctional $\mathrm{Fe}_{3} \mathrm{O}_{4}$-Ag core-shell nanostructures," Nano Research, vol. 2, no. 12, pp. 955-965, 2009. 
[73] P. K. Stoimenov, R. L. Klinger, G. L. Marchin, and K. J. Klabunde, "Metal oxide nanoparticles as bactericidal agents," Langmuir, vol. 18, no. 17, pp. 6679-6686, 2002.

[74] S. Ghosh, A. Upadhay, K. Abhishek, and A. Kumar, "Investigation of antimicrobial activity of silver nano particle loaded cotton fabrics which may promote wound healing," International Journal of Pharma and Bio Sciences, vol. 1, no. 3, article 32, 2010. 

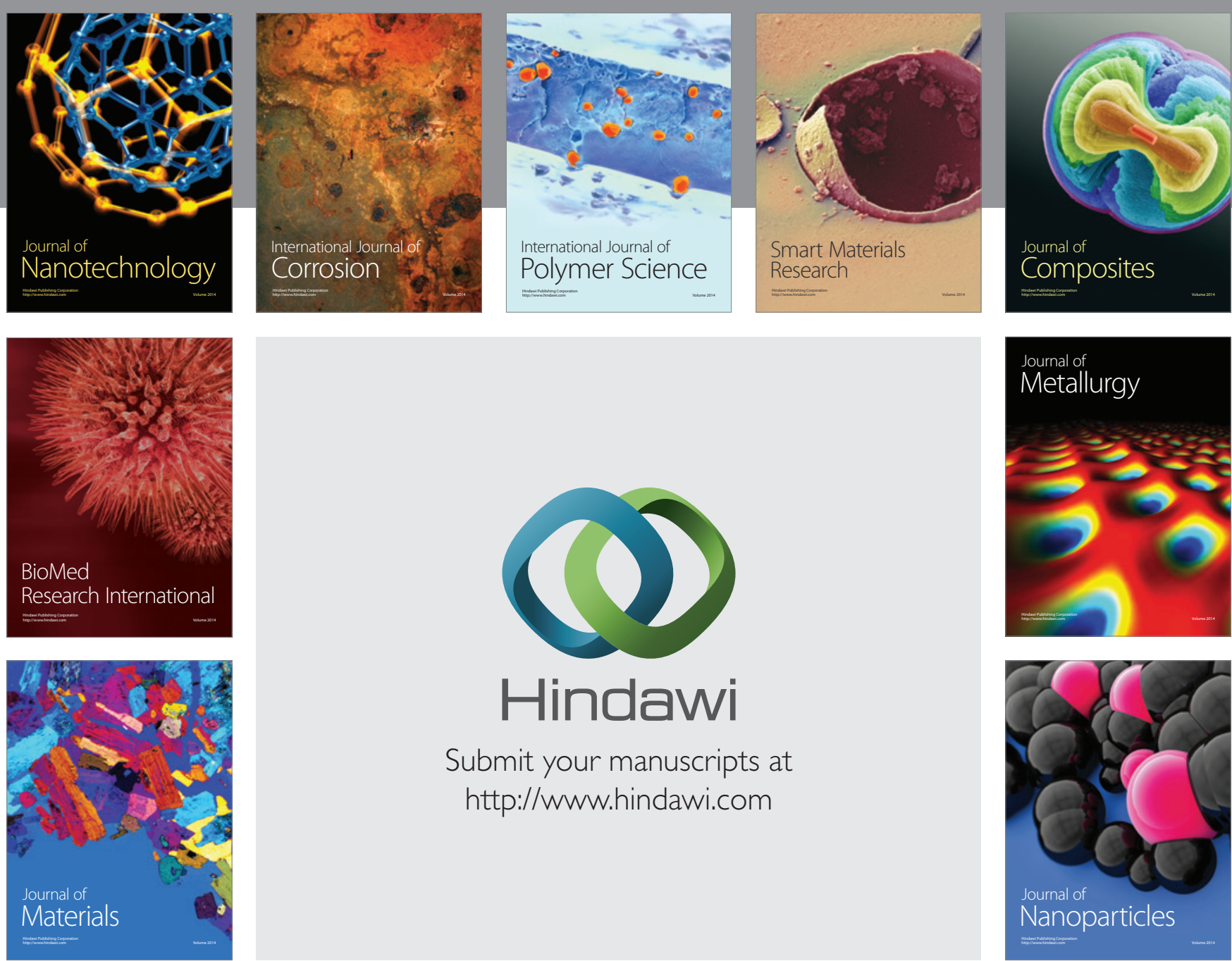

\section{Hindawi}

Submit your manuscripts at

http://www.hindawi.com

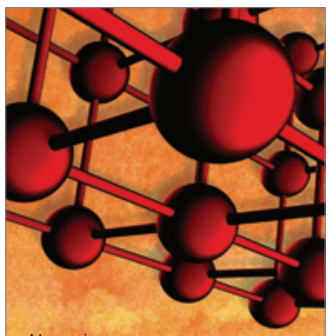

Materials Science and Engineering
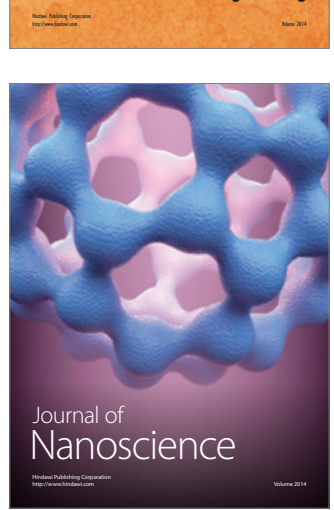
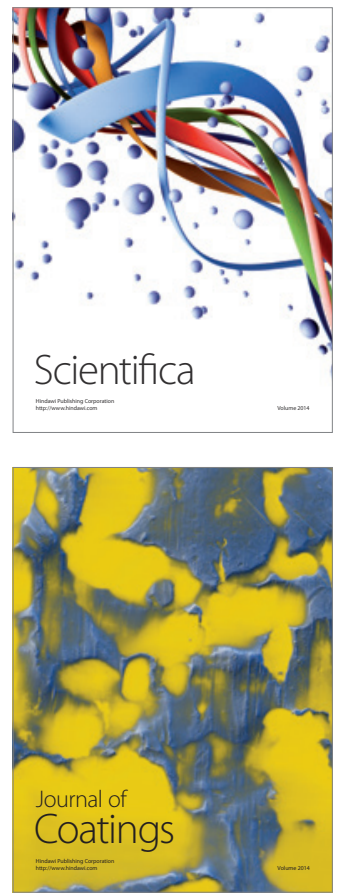
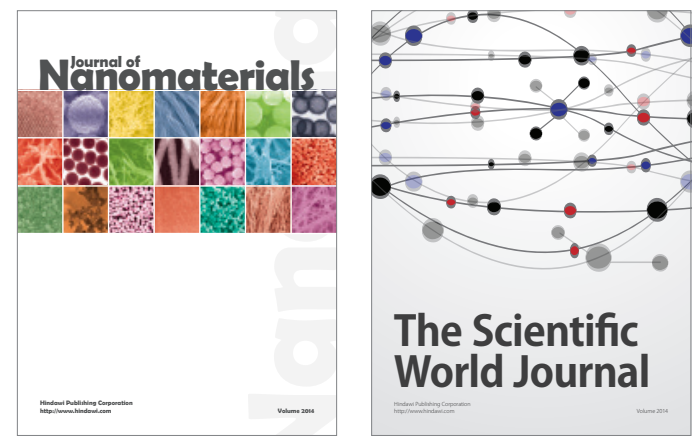

The Scientific World Journal
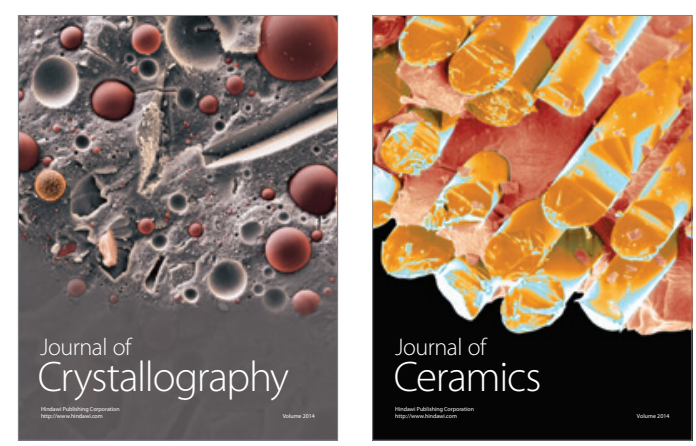
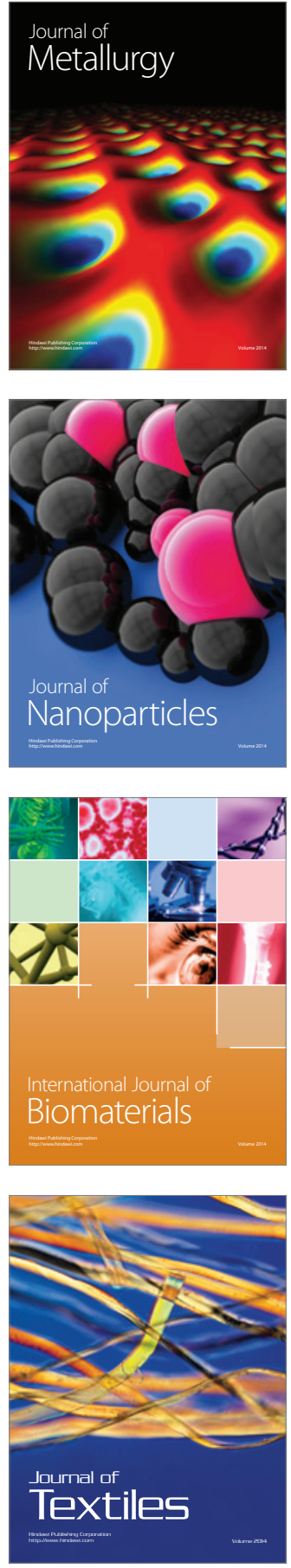